\title{
Triflic acid-catalyzed additions of 2-alkoxycarbonyl allylboronates to aldehydes. Study of scope and mechanistic investigation of the reaction stereochemistry.
}

\author{
Tim G. Elford, Yuichiro Arimura, Siu Hong Yu, Dennis G. Hall* \\ Department of Chemistry, University of Alberta, Edmonton, Alberta, Canada, T6G $2 G 2$
}

Supplementary Information

1. General Information

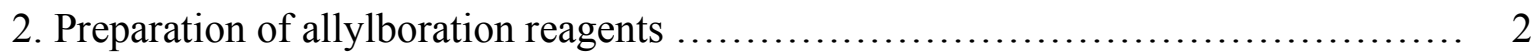

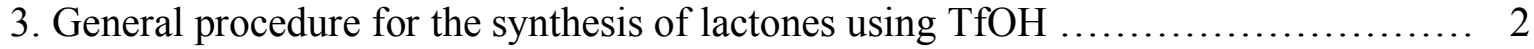

4. General procedure for the synthesis of lactones using thermal conditions followed by acidic conditions ............................................................ 10

5. General procedure for the synthesis of butyric acid methyl esters ................. 17

6. Synthesis of ${ }^{18} \mathrm{O}$ labeled aldehydes .............................................. 18

7. Cycle NOE values for lactones ................................................ 19

8. Table of ${ }^{1} \mathrm{H}$ NMR pattern for proton chemical shift of $\mathrm{H}^{4}$ and $\mathrm{H}^{5} \ldots \ldots \ldots \ldots \ldots \ldots \ldots \ldots . \ldots \ldots$

9. Copies of ${ }^{1} \mathrm{H}$ NMR and ${ }^{13} \mathrm{C}$ NMR for synthesized compounds ........................ 21

\section{General Information}

Unless otherwise noted, all reactions were performed under an argon atmosphere using flamedried glassware. Toluene, HMPA and $\mathrm{CH}_{2} \mathrm{Cl}_{2}$ were distilled over $\mathrm{CaH}_{2}$. THF was distilled over sodium/benzophenone ketyl. $\mathrm{NH}_{4} \mathrm{Cl}(\mathrm{aq})$ and $\mathrm{NaHCO}_{3}$ (aq) refer to saturated aqueous solutions. All aldehydes were purified by Kugelrohr distillation prior to use. Methyllithium was titrated according to the Gilman double titration method. ${ }^{1}$ Iodomethaneboronate ${ }^{2}$ and chloromethaneboronate ${ }^{3}$ were prepared according to literature procedures. TfOH was stored under $\mathrm{Ar}$ in a pear-shaped flask with a glass stopper and placed in a jar filled with anhydrous calcium sulfate which was then stored at $0{ }^{\circ} \mathrm{C}$. All other chemicals were used as received from commercial sources. Thin layer chromatography (TLC) was performed on silica gel plates and was visualized with UV light or potassium permanganate stain. NMR spectra were recorded on $300,400,500$ or $600 \mathrm{MHz}$ instruments. The residual solvent protons $\left({ }^{1} \mathrm{H}\right)$ or the solvent carbons $\left({ }^{13} \mathrm{C}\right)$ were used as internal standards ${ }^{1} \mathrm{H}$ NMR data are presented as follows: chemical shift in ppm downfield from tetramethylsilane (multiplicity, integration, coupling constant). The following abbreviations are used in reporting NMR data: s, singlet; br. s, broad singlet; $d$, doublet; t, triplet; br. t, broad triplet; app. t, apparent triplet; br. dt, broad doublet of triplets; $q$, quartet; dd, doublet of doublets; ddd, doublet of doublet of doublets; ddddd, doublet of doublet of doublet of doublet of doublets; ddq, doublet of doublet of quartets; dqd, doublet of quartet of doublets; dt, doublet of triplets; pt, pentet of triplets; qt, quartet of triplets; m, multiplet; sept, septet. High resolution mass spectra were recorded by the University of Alberta Mass 
Spectrometry Services Laboratory using either electron impact (EI) or electrospray (ES) ionization techniques. Infrared spectra were recorded by the University of Alberta Spectral Services and combustion analyses were performed by the University of Alberta Micro-Analytical Lab.

\section{Preparation of methyl (2E)-2-[4,4,5,5-tetramethyl-1,3,2-dioxaborolan-2-yl)methyl]-but-} 2-enoate $(E-1)$

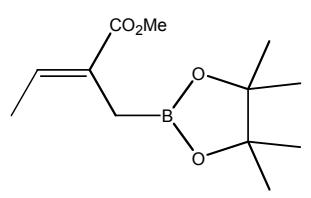

This compound was synthesized using known literature procedures and the product possessed identical spectroscopic characteristics to those reported in the literature. ${ }^{4}$

Preparation of methyl (2Z)-2-[4,4,5,5-tetramethyl-1,3,2-dioxaborolan-2-yl)methyl]-but-2enoate $(Z-1)$

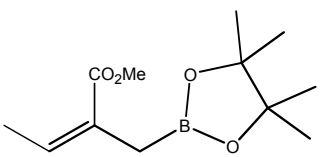

This compound was synthesized using known literature procedures and possessed identical spectroscopic characteristics to those reported in the literature. ${ }^{4}$ This compound could be isolated as a 20:1 mixture of $\mathrm{Z} / \mathrm{E}$ isomers, with the desired compound being the major component.

Preparation of isopropyl (2E)-2-[4,4,5,5-tetramethyl-1,3,2-dioxaborolan-2-yl)methyl]-but-2enoate $(E-26)$

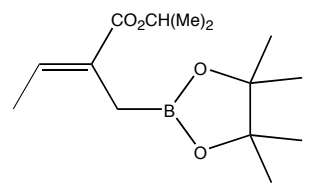

This compound was prepared using the identical procedure ${ }^{4}$ as that used to synthesize $E$-1 except that isopropyl propiolate was used instead of methyl propiolate as the alkyne source. Isopropyl propiolate was made using a known literature procedure. ${ }^{5}$

Flash chromatography ( $15 \%$ ethyl ether in hexanes) yielded the product as a pale yellow oil in $38 \%$ yield. ${ }^{1} \mathrm{H}$ NMR $\left(400 \mathrm{MHz}, \mathrm{CDCl}_{3}\right): \delta 6.83-6.76(\mathrm{~m}, 1 \mathrm{H}), 5.03(\mathrm{sept}, 1 \mathrm{H}, J=6.2 \mathrm{~Hz}), 1.85$ (br. s, 2H), 1.77 (br. dt, 3H, $J=7.0,0.9 \mathrm{~Hz}), 1.26$ (d, 6H, $J=6.2 \mathrm{~Hz}), 1.24$ (s, 12H); ${ }^{13} \mathrm{C}$ NMR $\left(100 \mathrm{MHz}, \mathrm{CDCl}_{3}\right): \delta 167.7,134.9,130.9,83.3,67.7,24.8,24.5,22.0,14.5 ;{ }^{11} \mathrm{~B}$ NMR $(128$ $\left.\mathrm{MHz}, \mathrm{CDCl}_{3}\right): \delta 31.7$; IR $\left(\mathrm{CH}_{2} \mathrm{Cl}_{2}\right.$ microscope, $\left.\mathrm{cm}^{-1}\right): 2979,2935,1704,1348,1325,1273,1146$; HRMS (EI, m/z) Calcd for $\mathrm{C}_{14} \mathrm{H}_{25}{ }^{11} \mathrm{BO}_{4}$ : 268.18460. Found: 268.18433. 


\section{General procedure for the synthesis of lactones under TfOH catalyzed conditions using the $E$-allylboronate ${ }^{4}$}

A solution of $E$-allylboronate $(100 \mathrm{mg}, 0.42 \mathrm{mmol})$ and aldehyde $(0.83 \mathrm{mmol})$ in toluene $(1 \mathrm{~mL})$ at $0{ }^{\circ} \mathrm{C}$ was treated with $\mathrm{TfOH}(4 \mu \mathrm{L}, 0.04 \mathrm{mmol})$ and stirred at $0{ }^{\circ} \mathrm{C}$ under an $\mathrm{Ar}$ atmosphere for $16 \mathrm{~h}$. The mixture was then diluted with $\mathrm{NH}_{4} \mathrm{Cl}(\mathrm{aq}) / \mathrm{NH}_{4} \mathrm{OH}(9: 1 \mathrm{v} / \mathrm{v}, 10 \mathrm{~mL})$ and extracted with $\mathrm{Et}_{2} \mathrm{O}(3 \times 20 \mathrm{~mL})$. The combined extracts were washed with brine $(2 \times 20 \mathrm{~mL})$, dried with anhydrous $\mathrm{Na}_{2} \mathrm{SO}_{4}$, filtered and concentrated. Crude products were then purified by flash chromatography to yield the corresponding lactone.

\section{Synthesis of cis-4-Methyl-3-methylene-5-(4-nitro-phenyl)-dihydro-furan-2-one (10a)}

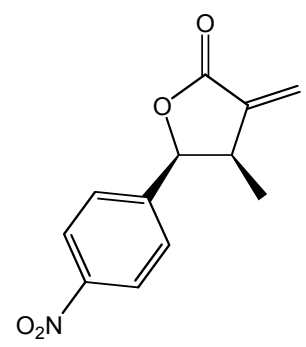

Flash chromatography (20\% EtOAc/Hexanes) yielded the product as a yellow solid in $53 \%$ yield. ${ }^{1} \mathrm{H}$ NMR $\left(400 \mathrm{MHz}, \mathrm{CDCl}_{3}\right)$ : $\delta 8.27-8.25(\mathrm{~m}, 2 \mathrm{H}), 7.41-7.38(\mathrm{~m}, 2 \mathrm{H}), 6.38(\mathrm{~d}, 1 \mathrm{H}, J=2.5$ $\mathrm{Hz}), 5.70(\mathrm{~d}, 1 \mathrm{H}, J=8.1 \mathrm{~Hz}), 5.66(\mathrm{~d}, 1 \mathrm{H}, J=2.4 \mathrm{~Hz}), 3.57-3.47(\mathrm{~m}, 1 \mathrm{H}), 0.80(\mathrm{~d}, 3 \mathrm{H}, J=7.2$ $\mathrm{Hz}) ;{ }^{13} \mathrm{C}$ NMR $\left(100 \mathrm{MHz}, \mathrm{CDCl}_{3}\right): \delta 169.7,147.9,143.6,135.2,126.9,123.8,122.9,80.7,38.7$, 15.8; IR $\left(\mathrm{CH}_{2} \mathrm{Cl}_{2}\right.$ cast film, $\left.\mathrm{cm}^{-1}\right)$ : 3082, 2973, 2933, 1770, 1521, 1349; HRMS (EI, m/z) Calcd for $\mathrm{C}_{12} \mathrm{H}_{11} \mathrm{NO}_{4}$ : 233.06880. Found: 233.06846. Elem. Anal. (\%) Calcd for $\mathrm{C}_{12} \mathrm{H}_{11} \mathrm{NO}_{4}$ : C, 61.80; H, 4.75; N, 6.01. Found: C, 61.99; H, 4.97; N, 5.74.

\section{Synthesis of trans-5-(4-Bromo-phenyl)-4-methyl-3-methylene-dihydro-furan-2-one (11b)}

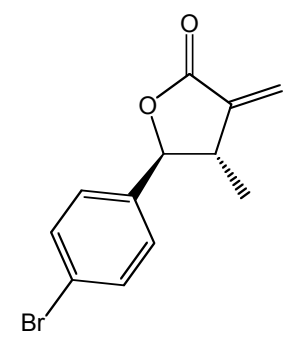

Flash chromatography (20\% EtOAc/Hexanes) yielded the product as a white solid in $62 \%$ yield. ${ }^{1} \mathrm{H}$ NMR $\left(500 \mathrm{MHz}, \mathrm{CDCl}_{3}\right): \delta 7.58-7.52(\mathrm{~m}, 2 \mathrm{H}), 7.25-7.21(\mathrm{~m}, 2 \mathrm{H}), 6.33(\mathrm{~d}, 1 \mathrm{H}, J=3.3$ $\mathrm{Hz}), 5.60(\mathrm{~d}, 1 \mathrm{H}, J=2.9 \mathrm{~Hz}), 4.87(\mathrm{~d}, 1 \mathrm{H}, J=7.7 \mathrm{~Hz}), 2.94-2.86(\mathrm{~m}, 1 \mathrm{H}), 1.33(\mathrm{~d}, 3 \mathrm{H}, J=6.7$ $\mathrm{Hz}) ;{ }^{13} \mathrm{C}$ NMR $\left(100 \mathrm{MHz}, \mathrm{CDCl}_{3}\right): \delta 169.6,139.9,137.3,131.9,127.4,122.7,121.2,85.0,43.3$, 15.7; IR $\left(\mathrm{CH}_{2} \mathrm{Cl}_{2}\right.$ cast film, cm $\left.{ }^{-1}\right): 2968,2931,1769,1490,1254,1039 ; \mathrm{HRMS}$ (EI, m/z) Calcd for $\mathrm{C}_{12} \mathrm{H}_{11} \mathrm{O}_{2}{ }^{81} \mathrm{Br}$ : 267.99219. Found: 267.99251. Elem. Anal. (\%) Calcd for $\mathrm{C}_{12} \mathrm{H}_{11} \mathrm{O}_{2}{ }^{81} \mathrm{Br}$ : $\mathrm{C}$, 53.96; H, 4.15. Found: C, 53.85; H, 4.48. 


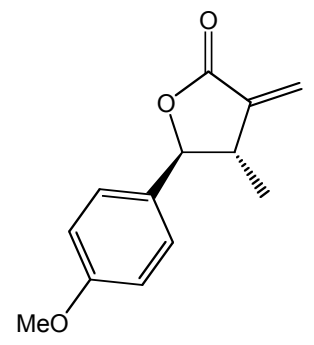

Flash chromatography (20\% EtOAc/Hexanes) yielded the product as a pale yellow solid in $62 \%$ yield. Spectral data for $\mathbf{1 2 b}$ was identical to that found in literature. ${ }^{6}$

\section{Synthesis of trans-4-Methyl-3-methylene-5-o-tolyl-dihydro-furan-2-one (13b)}

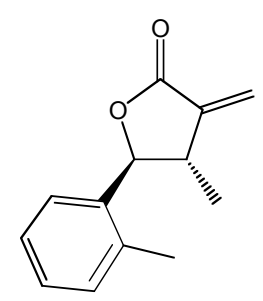

Flash chromatography (10\% EtOAc/Hexanes) yielded the product as a pale yellow oil in $49 \%$ yield. ${ }^{1} \mathrm{H}$ NMR $\left(500 \mathrm{MHz}, \mathrm{CDCl}_{3}\right): \delta 7.30-7.18(\mathrm{~m}, 4 \mathrm{H}), 6.33(\mathrm{~d}, 1 \mathrm{H}, J=3.0 \mathrm{~Hz}), 5.60(\mathrm{~d}, 1 \mathrm{H}, J$ $=2.7 \mathrm{~Hz}), 5.23(\mathrm{~d}, 1 \mathrm{H}, J=7.1 \mathrm{~Hz}), 3.10-3.02(\mathrm{~m}, 1 \mathrm{H}), 2.37(\mathrm{~s}, 3 \mathrm{H}), 1.32(\mathrm{~d}, 3 \mathrm{H}, J=6.9 \mathrm{~Hz})$; ${ }^{13} \mathrm{C}$ NMR $\left(125 \mathrm{MHz}, \mathrm{CDCl}_{3}\right): \delta 170.4,140.6,136.5,135.5,130.9,128.6,126.6,125.7,121.3$, 83.1, 42.6, 19.5, 17.2; IR (microscope, $\mathrm{cm}^{-1}$ ): 2968, 1768, 1312, 1255, 1140, 986; HRMS (EI, $\mathrm{m} / \mathrm{z}$ ) Calcd for $\mathrm{C}_{13} \mathrm{H}_{14} \mathrm{O}_{2}: 202.09938$. Found: 202.09908 .

\section{Synthesis of trans-4-Methyl-3-methylene-5-p-tolyl-dihydro-furan-2-one (14b)}

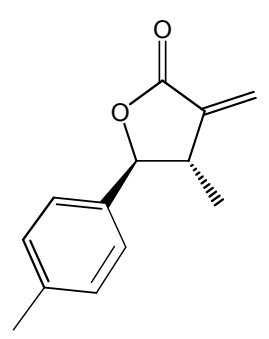

Flash chromatography (20\% EtOAc/Hexanes) yielded the product as a pale yellow oil in $59 \%$ yield. ${ }^{1} \mathrm{H}$ NMR $\left(500 \mathrm{MHz}, \mathrm{CDCl}_{3}\right): \delta 7.27-7.19(\mathrm{~m}, 4 \mathrm{H}), 6.31(\mathrm{~d}, 1 \mathrm{H}, J=3.2 \mathrm{~Hz}), 5.58(\mathrm{~d}, 1 \mathrm{H}$, $J=2.9 \mathrm{~Hz}), 4.87(\mathrm{~d}, 1 \mathrm{H}, J=7.8 \mathrm{~Hz}), 2.99-2.90(\mathrm{~m}, 1 \mathrm{H}), 2.37$ (s, 3H), $1.31(\mathrm{~d}, 3 \mathrm{H}, J=6.9 \mathrm{~Hz})$; ${ }^{13} \mathrm{C} \mathrm{NMR}\left(100 \mathrm{MHz}, \mathrm{CDCl}_{3}\right): \delta 170.2,140.8,138.8,135.4,129.5,126.0,120.8,86.0,43.4,21.2$, 15.8; IR $\left(\mathrm{CH}_{2} \mathrm{Cl}_{2}\right.$ cast film, $\left.\mathrm{cm}^{-1}\right)$ : 2968, 2925, 1769, 1256, 1138, 983; HRMS (EI, m/z) Calcd for $\mathrm{C}_{13} \mathrm{H}_{14} \mathrm{O}_{2}$ : 202.09938. Found: 202.09955. 


\section{Synthesis of trans-4-methyl-3-methylene-5-phenyl-dihydro-furan-2-one (15b)}

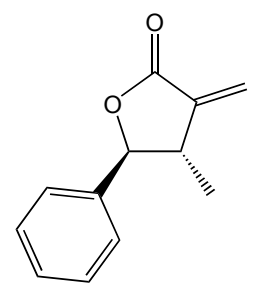

Flash chromatography (15\% EtOAc/Hexanes) yielded the product as a pale yellow solid in 58\% yield. Spectral data for $\mathbf{1 5 b}$ was identical to that found in literature. ${ }^{7}$

\section{Synthesis of trans-4-Methyl-3-methylene-5-propenyl-dihydro-furan-2-one (16b)}

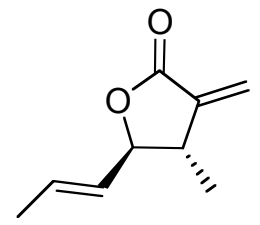

Flash chromatography (20\% EtOAc/Hexanes) yielded the product as a colorless oil in $79 \%$ yield. ${ }^{1} \mathrm{H}$ NMR $\left(500 \mathrm{MHz}, \mathrm{CDCl}_{3}\right): \delta 6.23(\mathrm{~d}, 1 \mathrm{H}, J=3.3 \mathrm{~Hz}), 5.88(\mathrm{dqd}, 1 \mathrm{H}, J=15.2,6.7,0.9 \mathrm{~Hz})$, $5.52(\mathrm{~d}, 1 \mathrm{H}, J=3.0 \mathrm{~Hz}), 5.51(\mathrm{ddq}, 1 \mathrm{H}, J=15.3,7.9,1.5 \mathrm{~Hz}), 4.30$ (t, $1 \mathrm{H}, J=7.9 \mathrm{~Hz}), 2.78-$ $2.68(\mathrm{~m}, 1 \mathrm{H}), 1.77(\mathrm{ddd}, 3 \mathrm{H}, J=6.6,1.7,0.6 \mathrm{~Hz}), 1.23(\mathrm{~d}, 3 \mathrm{H}, J=6.9 \mathrm{~Hz}) ;{ }^{13} \mathrm{C} \mathrm{NMR}(100 \mathrm{MHz}$, $\left.\mathrm{CDCl}_{3}\right): \delta 170.0,140.6,131.9,127.9,120.4,85.6,40.9,17.9,15.5 ; \mathrm{IR}\left(\mathrm{CH}_{2} \mathrm{Cl}_{2}\right.$ cast film, $\left.\mathrm{cm}^{-1}\right)$ : 3096, 2969, 2920, 1766, 1248, 1143, 966; HRMS (EI, m/z) Calcd for $\mathrm{C}_{9} \mathrm{H}_{12} \mathrm{O}_{2}$ : 152.08372 . Found: 152.08388.

\section{Synthesis of cis-5-Hept-1-ynyl-4-methyl-3-methylene-dihydro-furan-2-one (17a)}

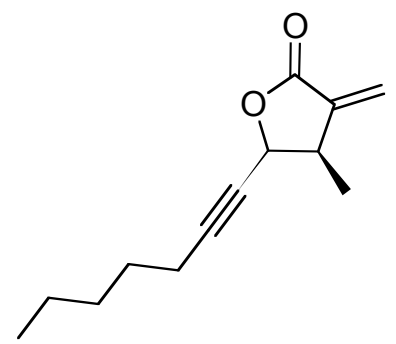

Flash chromatography (20\% EtOAc/Hexanes) yielded the product as an orange oil in $63 \%$ yield. ${ }^{1} \mathrm{H}$ NMR $\left(600 \mathrm{MHz}, \mathrm{CDCl}_{3}\right): \delta 6.27(\mathrm{~d}, 1 \mathrm{H}, J=2.9 \mathrm{~Hz}), 5.57(\mathrm{~d}, 1 \mathrm{H}, J=2.7 \mathrm{~Hz}), 5.23(\mathrm{dt}, 1 \mathrm{H}, J$ $=8.0,2.1 \mathrm{~Hz}), 3.22-3.15(\mathrm{~m}, 1 \mathrm{H}), 2.23(\mathrm{td}, 2 \mathrm{H}, J=7.2,2.1 \mathrm{~Hz}), 1.54-1.48(\mathrm{~m}, 2 \mathrm{H}), 1.38-$ $1.27(\mathrm{~m}, 4 \mathrm{H}), 1.31(\mathrm{~d}, 3 \mathrm{H}, J=7.0 \mathrm{~Hz}), 0.89(\operatorname{app~t}, 3 \mathrm{H}, J=7.1 \mathrm{~Hz}) ;{ }^{13} \mathrm{C} \mathrm{NMR}(125 \mathrm{MHz}$, $\left.\mathrm{CDCl}_{3}\right): \delta 169.7,139.3,121.6,91.4,73.8,72.2,37.9,31.0,28.0,22.1,18.7,15.1,14.0$; IR $\left(\mathrm{CH}_{2} \mathrm{Cl}_{2}\right.$ cast film, $\left.\mathrm{cm}^{-1}\right): 2957,2933,2861,2233,1771,1244,1116,969 ; \mathrm{HRMS}$ (EI, m/z) Calcd for $\mathrm{C}_{13} \mathrm{H}_{18} \mathrm{O}_{2}$ : 206.13068. Found: 206.13048. Elem. Anal. (\%) Calcd for $\mathrm{C}_{13} \mathrm{H}_{18} \mathrm{O}_{2}: \mathrm{C}, 75.69 ; \mathrm{H}$, 8.80. Found: C, 75.26; H, 8.99. 


\section{Synthesis of cis-4-Methyl-3-methylene-5-phenethyl-dihydro-furan-2-one (18a)}

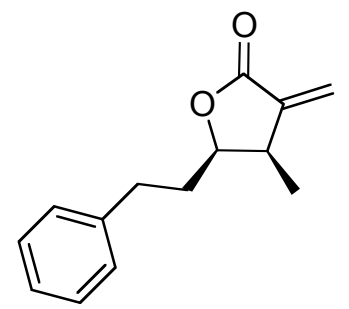

Flash chromatography (20\% EtOAc/Hexanes) yielded the product as a colorless oil in $46 \%$ yield. ${ }^{1} \mathrm{H}$ NMR $\left(500 \mathrm{MHz}, \mathrm{CDCl}_{3}\right): \delta 7.35-7.30(\mathrm{~m}, 2 \mathrm{H}), 7.26-7.21(\mathrm{~m}, 3 \mathrm{H}), 6.25(\mathrm{~d}, 1 \mathrm{H}, J=2.9$ $\mathrm{Hz}), 5.56(\mathrm{~d}, 1 \mathrm{H}, J=2.6 \mathrm{~Hz}), 4.57-4.51(\mathrm{~m}, 1 \mathrm{H}), 3.23-3.12(\mathrm{~m}, 1 \mathrm{H}), 2.94(\mathrm{ddd}, 1 \mathrm{H}, J=19.7$, 8.7, $5.8 \mathrm{~Hz}$ ), 2.74 (ddd, $1 \mathrm{H}, J=16.7,8.5,8.5 \mathrm{~Hz}), 1.89-1.78(\mathrm{~m}, 2 \mathrm{H}), 1.18$ (d, 3H, $J=7.2 \mathrm{~Hz}$ ); ${ }^{13} \mathrm{C} \mathrm{NMR}\left(125 \mathrm{MHz}, \mathrm{CDCl}_{3}\right): \delta 170.4,141.0,140.8,128.6,128.6,126.2,120.9,80.0,37.5,32.8$, 31.9, 13.9; IR $\left(\mathrm{CH}_{2} \mathrm{Cl}_{2}\right.$ cast film, $\left.\mathrm{cm}^{-1}\right): 3506,3062,3027,2968,1762,1267,1245,1124$; HRMS (EI, m/z) Calcd for $\mathrm{C}_{14} \mathrm{H}_{16} \mathrm{O}_{2}: 216.11504$. Found: 216.11533. Elem. Anal. (\%) Calcd for $\mathrm{C}_{14} \mathrm{H}_{16} \mathrm{O}_{2}$ : C, 77.75; H, 7.46. Found: C, 77.99; H, 7.56.

\section{Synthesis of cis-5-Cyclohexyl-4-methyl-3-methylene-dihydro-furan-2-one (19a)}

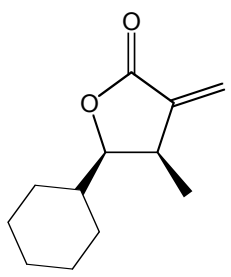

Flash chromatography $(10 \%$ EtOAc/Hexanes $)$ yielded the product as a clear oil in $11 \%$ yield. ${ }^{1} \mathrm{H}$ NMR $\left(600 \mathrm{MHz}, \mathrm{CDCl}_{3}\right): \delta 6.13(\mathrm{~d}, 1 \mathrm{H}, J=2.3 \mathrm{~Hz}), 5.53(\mathrm{~d}, 1 \mathrm{H}, J=2.0 \mathrm{~Hz}), 4.14(\mathrm{t}, 1 \mathrm{H}, J=$ $7.0 \mathrm{~Hz}), 3.13$ (pt, 1H, J=7.2, $2.2 \mathrm{~Hz}), 1.93-1.85(\mathrm{~m}, 1 \mathrm{H}), 1.82-1.72(\mathrm{~m}, 2 \mathrm{H}), 1.71-1.65(\mathrm{~m}$, $1 \mathrm{H}), 1.65-1.58(\mathrm{~m}, 2 \mathrm{H}), 1.33-1.00(\mathrm{~m}, 5 \mathrm{H}), 1.16(\mathrm{~d}, 3 \mathrm{H}, J=7.2 \mathrm{~Hz}) ;{ }^{13} \mathrm{C} \mathrm{NMR}(100 \mathrm{MHz}$, $\left.\mathrm{CDCl}_{3}\right): \delta 170.7,142.1,119.7,85.0,38.2,37.4,29.3,28.8,26.2,25.8,25.5,14.5$; IR (microscope, $\mathrm{cm}^{-1}$ ): 2930, 2854, 1765, 1268, 1151, 963; HRMS (EI, m/z) Calcd for $\mathrm{C}_{12} \mathrm{H}_{18} \mathrm{O}_{2}$ : 194.13068. Found: 194.13038.

General procedure for the synthesis of lactones under TfOH catalyzed conditions using the $Z$-allylboronate ${ }^{4}$

The procedure for the $\mathrm{TfOH}$ catalyzed allylboration reaction using the $Z$-allylboronate was identical to that utilized in the reaction using the $E$-allylboronate. 


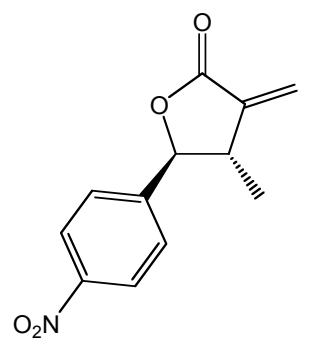

Flash chromatography (20\% EtOAc/Hexanes) yielded the product as a pale yellow solid in $52 \%$ yield. ${ }^{1} \mathrm{H}$ NMR $\left(400 \mathrm{MHz}, \mathrm{CDCl}_{3}\right): \delta 8.30-8.26(\mathrm{~m}, 2 \mathrm{H}), 7.57-7.52(\mathrm{~m}, 2 \mathrm{H}), 6.38(\mathrm{~d}, 1 \mathrm{H}, J=$ $3.3 \mathrm{~Hz}), 5.65(\mathrm{~d}, 1 \mathrm{H}, J=2.7 \mathrm{~Hz}), 5.02(\mathrm{~d}, 1 \mathrm{H}, J=7.6 \mathrm{~Hz}), 2.96-2.86(\mathrm{~m}, 1 \mathrm{H}), 1.40(\mathrm{~d}, 3 \mathrm{H}, J=$ $6.8 \mathrm{~Hz}) ;{ }^{13} \mathrm{C} \mathrm{NMR}\left(100 \mathrm{MHz}, \mathrm{CDCl}_{3}\right): \delta 169.3,145.7,139.3,126.5,124.2,122.2,94.4,84.3$, 43.5, 16.2; IR (microscope, $\mathrm{cm}^{-1}$ ): 3510, 3114, 2974, 2936, 1773, 1519, 1352, 1266, 1145; HRMS (EI, m/z) Calcd for $\mathrm{C}_{12} \mathrm{H}_{11} \mathrm{NO}_{4}$ : 233.06880. Found: 233.06893. Elem. Anal. (\%) Calcd for $\mathrm{C}_{12} \mathrm{H}_{11} \mathrm{NO}_{4}$ : C, 61.84; H, 4.76; N, 6.01. Found: C, 61.91; H, 4.83; N, 5.99.

\section{Synthesis of trans-5-(4-Bromo-phenyl)-4-methyl-3-methylene-dihydro-furan-2-one (11b)}

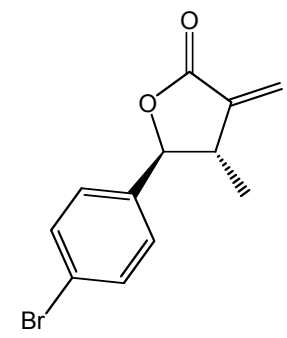

Flash chromatography (20\% EtOAc/Hexanes) yielded the product as a white solid in 59\% yield. Spectral data for this compound was identical to that reported when the $E$-allylboronate and corresponding aldehyde were reacted under $\mathrm{TfOH}$ acid conditions.

Synthesis of trans-5-(4-Methoxy-phenyl)-4-methyl-3-methylene-dihydro-furan-2-one (12b)

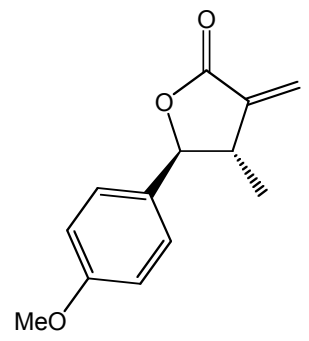

Flash chromatography (20\% EtOAc/Hexanes) yielded the product as a pale yellow solid in $40 \%$ yield. Spectral data for $\mathbf{1 2 b}$ was identical to that found in literature. ${ }^{5}$ 


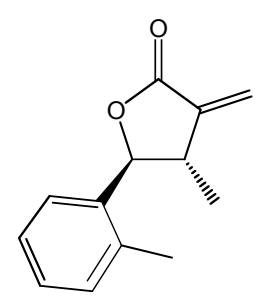

Flash chromatography (15\% EtOAc/Hexanes) yielded the product as a pale yellow oil in $49 \%$ yield. Spectral data for this compound was identical to that reported when the $E$-allylboronate and corresponding aldehyde were reacted under $\mathrm{TfOH}$ acid conditions.

Synthesis of trans-4-Methyl-3-methylene-5-p-tolyl-dihydro-furan-2-one (14b)

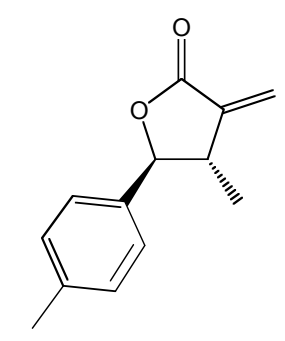

Flash chromatography (20\% EtOAc/Hexanes) yielded the product as a pale yellow oil in $91 \%$ yield. Spectral data for this compound was identical to that reported when the $E$-allylboronate and corresponding aldehyde were reacted under $\mathrm{TfOH}$ acid conditions.

Synthesis of trans-4-methyl-3-methylene-5-phenyl-dihydro-furan-2-one (15b)

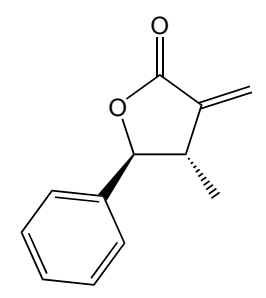

Flash chromatography (15\% EtOAc/Hexanes) yielded the product as a pale yellow solid in $78 \%$ yield. Spectral data for $\mathbf{1 5 b}$ was identical to that found in literature. ${ }^{7}$

Synthesis of trans-4-Methyl-3-methylene-5-propenyl-dihydro-furan-2-one (16b)<smiles>C=C1C(=O)OC(/C=C/C)[C@@H]1C</smiles> 
Flash chromatography (20\% EtOAc/Hexanes) yielded the product as a colorless oil in $26 \%$ yield. (Note: Compound is relatively volatile, and some product was lost during solvent removal. This can be reduced by keep the temperature on the rotovap at a temperature less than $30{ }^{\circ} \mathrm{C}$.) Spectral data for this compound was identical to that reported when the $E$-allylboronate and corresponding aldehyde were reacted under $\mathrm{TfOH}$ acid conditions.

\section{Synthesis of trans-5-Hept-1-ynyl-4-methyl-3-methylene-dihydro-furan-2-one (17b)}

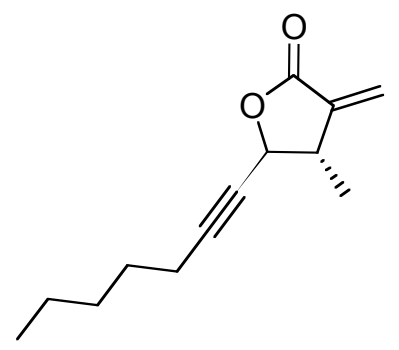

Flash chromatography (20\% EtOAc/Hexanes) yielded the product as a pale yellow oil in $46 \%$ yield. (Note: The cis diastereomer was also isolated in $17 \%$ yield.) ${ }^{1} \mathrm{H} \mathrm{NMR}\left(600 \mathrm{MHz}, \mathrm{CDCl}_{3}\right)$ : $\delta 6.25(\mathrm{~d}, 1 \mathrm{H}, J=3.0 \mathrm{~Hz}), 5.56(\mathrm{~d}, 1 \mathrm{H}, J=2.8 \mathrm{~Hz}), 4.57(\mathrm{dt}, 1 \mathrm{H}, J=7.2,1.9 \mathrm{~Hz}), 3.08-3.02$ $(\mathrm{m}, 1 \mathrm{H}), 2.24(\mathrm{td}, 2 \mathrm{H}, J=7.2,2.1 \mathrm{~Hz}), 1.56-1.50(\mathrm{~m}, 2 \mathrm{H}), 1.40-1.32(\mathrm{~m}, 4 \mathrm{H}), 1.31(\mathrm{~d}, 3 \mathrm{H}, J=$ $6.6 \mathrm{~Hz}), 0.91$ (app t, 3H, $J=7.2 \mathrm{~Hz}) ;{ }^{13} \mathrm{C} \mathrm{NMR}\left(125 \mathrm{MHz}, \mathrm{CDCl}_{3}\right)$ : $\delta 169.2,139.5,121.3,89.5$, 75.9, 74.0, 42.7, 31.0, 28.0, 22.2, 18.8, 16.2, 14.0; IR $\left(\mathrm{CH}_{2} \mathrm{Cl}_{2}\right.$ cast film, $\left.\mathrm{cm}^{-1}\right): 2933,2861,2241$, 1772, 1304, 1254, 1132, 977; HRMS (EI, m/z) Calcd for $\mathrm{C}_{13} \mathrm{H}_{18} \mathrm{O}_{2}$ : 206.13068. Found: 206.13126 .

\section{Synthesis of trans-4-Methyl-3-methylene-5-phenethyl-dihydro-furan-2-one (18b)}

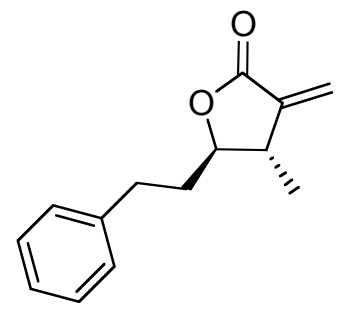

Flash chromatography (20\% EtOAc/Hexanes) yielded the product as a colorless oil in 44\% yield. ${ }^{1} \mathrm{H}$ NMR (400 MHz, $\left.\mathrm{CDCl}_{3}\right): \delta 7.33-7.18(\mathrm{~m}, 5 \mathrm{H}), 6.24(\mathrm{~d}, 1 \mathrm{H}, J=3.1 \mathrm{~Hz}), 5.54(\mathrm{~d}, 1 \mathrm{H}, J=$ $2.9 \mathrm{~Hz}), 3.99(\mathrm{ddd}, 1 \mathrm{H}, J=10.9,6.8,4.1 \mathrm{~Hz}), 2.91(\mathrm{ddd}, 1 \mathrm{H}, J=13.9,9.3,5.3 \mathrm{~Hz}), 2.80-2.66$ $(\mathrm{m}, 2 \mathrm{H}), 2.08-1.91(\mathrm{~m}, 2 \mathrm{H}), 1.22(\mathrm{~d}, 3 \mathrm{H}, J=6.9 \mathrm{~Hz}) ;{ }^{13} \mathrm{C} \mathrm{NMR}\left(125 \mathrm{MHz}, \mathrm{CDCl}_{3}\right): \delta 170.3$, $140.9,128.6,128.5,126.2,121.1,84.1,40.1,36.8,32.0,31.7,17.0 ; \mathrm{IR}\left(\mathrm{CH}_{2} \mathrm{Cl}_{2}\right.$ cast film, cm $\left.{ }^{-1}\right)$ : 3506, 3062, 3027, 2932, 1766, 1250, 1134, 701; HRMS (EI, m/z) Calcd for $\mathrm{C}_{14} \mathrm{H}_{16} \mathrm{O}_{2}$ : 216.11504. Found: 216.11483. 


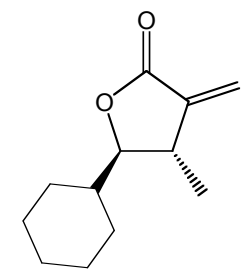

Flash chromatography (20\% EtOAc/Hexanes followed by a second column using 100\% dichloromethane) yielded the product as a colorless oil in $71 \%$ yield. ${ }^{1} \mathrm{H} \mathrm{NMR}(400 \mathrm{MHz}$, $\left.\mathrm{CDCl}_{3}\right): \delta 6.23(\mathrm{~d}, 1 \mathrm{H}, J=3.1 \mathrm{~Hz}), 5.55(\mathrm{~d}, 1 \mathrm{H}, J=2.7 \mathrm{~Hz}), 3.83(\mathrm{t}, 1 \mathrm{H}, J=5.6 \mathrm{~Hz}), 2.99-2.81$ $(\mathrm{m}, 1 \mathrm{H}), 1.90-1.66(\mathrm{~m}, 5 \mathrm{H}), 1.60-1.50(\mathrm{~m}, 1 \mathrm{H}), 1.30-1.10(\mathrm{~m}, 5 \mathrm{H}), 1.25(\mathrm{~d}, 3 \mathrm{H}, J=6.9 \mathrm{~Hz})$; ${ }^{13} \mathrm{C}$ NMR $\left(125 \mathrm{MHz}, \mathrm{CDCl}_{3}\right): \delta 170.2,141.2,121.0,89.0,42.4,36.8,28.6,28.0,26.4,26.0$, 25.8, 19.4; IR (neat film, $\mathrm{cm}^{-1}$ ): 2928, 2854, 1765, 1663, 1451, 1255, 1134, 991; HRMS (EI, $\mathrm{m} / \mathrm{z}$ ) Calcd for $\mathrm{C}_{12} \mathrm{H}_{18} \mathrm{O}_{2}$ : 194.13068. Found: 194.13077.

\section{General procedure for the synthesis of lactones under thermal conditions followed by $p$ - Toluenesulfonic acid monohydrate using the $E$-allylboronate ${ }^{4}$}

A solution of $E$-allylboronate $(100 \mathrm{mg}, 0.42 \mathrm{mmol})$ and aldehyde $(0.46 \mathrm{mmol})$ in toluene $(0.5$ $\mathrm{mL}$ ) was heated to $110^{\circ} \mathrm{C}$ in a high pressure vessel under an $\mathrm{Ar}$ atmosphere for $72 \mathrm{~h} . p-\mathrm{TSA} \cdot \mathrm{H}_{2} \mathrm{O}$ (230 $\mathrm{mg}, 1.2 \mathrm{mmol}$ ) was then added and the mixture was stirred overnight at room temperature. The reaction was quenched with $\mathrm{NaHCO}_{3}(\mathrm{aq})(20 \mathrm{~mL})$ and extracted with $\mathrm{Et}_{2} \mathrm{O}(3 \mathrm{x} 20 \mathrm{~mL})$. The combined extracts were washed with brine $(20 \mathrm{~mL})$, dried over anhydrous $\mathrm{Na}_{2} \mathrm{SO}_{4}$, filtered and concentrated. Flash chromatography gave the corresponding lactone.

Synthesis of cis-4-Methyl-3-methylene-5-(4-nitro-phenyl)-dihydro-furan-2-one (10a)

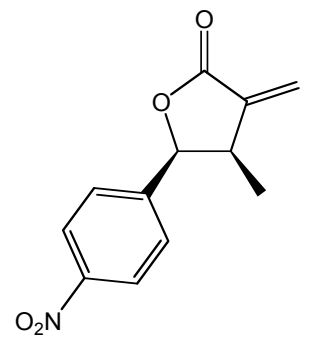

Flash chromatography $(20 \%$ EtOAc/Hexanes) yielded the product as a yellow solid in $90 \%$ yield. Spectral data for this compound was identical to that reported when the $E$-allylboronate and corresponding aldehyde were reacted under $\mathrm{TfOH}$ acid conditions. 


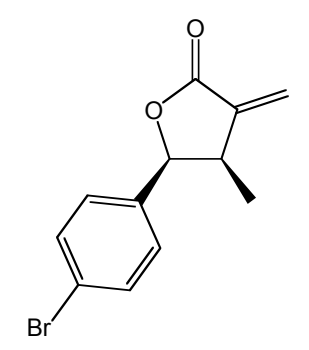

Flash chromatography (20\% EtOAc/Hexanes) yielded the product as a white solid in $79 \%$ yield. ${ }^{1} \mathrm{H}$ NMR (400 MHz, $\left.\mathrm{CDCl}_{3}\right): \delta 7.54-7.50(\mathrm{~m}, 2 \mathrm{H}), 7.09-7.05(\mathrm{~m}, 2 \mathrm{H}), 6.35(\mathrm{~d}, 1 \mathrm{H}, J=2.9$ $\mathrm{Hz}), 5.61(\mathrm{~d}, 1 \mathrm{H}, J=2.5 \mathrm{~Hz}), 5.59(\mathrm{~d}, 1 \mathrm{H}, J=8.0 \mathrm{~Hz}), 3.50-3.40(\mathrm{~m}, 1 \mathrm{H}), 0.82(\mathrm{~d}, 3 \mathrm{H}, J=7.1$ $\mathrm{Hz}) ;{ }^{13} \mathrm{C}$ NMR $\left(100 \mathrm{MHz}, \mathrm{CDCl}_{3}\right): \delta 170.3,139.7,135.4,131.8,127.7,122.5,122.2,81.4,38.8$, 15.5; IR (microscope, $\mathrm{cm}^{-1}$ ): 3096, 2973, 2933, 1768, 1491, 1247, 1148, 996; HRMS (EI, m/z) Calcd for $\mathrm{C}_{12} \mathrm{H}_{11} \mathrm{O}_{2}{ }^{81} \mathrm{Br}$ : 267.99219. Found: 267.99262. Elem. Anal. (\%) Calcd for $\mathrm{C}_{12} \mathrm{H}_{11} \mathrm{O}_{2}{ }^{81} \mathrm{Br}$ : C, 53.96; H, 4.15. Found: C, 54.32; H, 4.26.

\section{Synthesis of cis-5-(4-Methoxy-phenyl)-4-methyl-3-methylene-dihydro-furan-2-one (12a)}

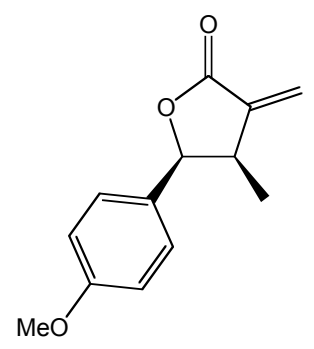

Flash chromatography (20\% EtOAc/Hexanes) yielded the product as a pale yellow solid in 31\% yield. (Note: The trans diastereomer was also isolated in 31\% yield.) Spectral data for 12a was identical to that found in literature. ${ }^{5}$

\section{Synthesis of cis-4-Methyl-3-methylene-5-o-tolyl-dihydro-furan-2-one (13a)}

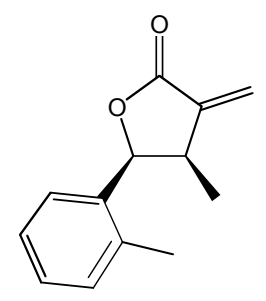

Flash chromatography (15\% EtOAc/Hexanes) yielded the product as a yellow solid in $80 \%$ yield. ${ }^{1} \mathrm{H}$ NMR $\left(500 \mathrm{MHz}, \mathrm{CDCl}_{3}\right): \delta$ 7.31-7.17 (m, 4H), $6.36(\mathrm{~d}, 1 \mathrm{H}, J=2.4 \mathrm{~Hz}), 5.83(\mathrm{~d}, 1 \mathrm{H}, J=7.7$ $\mathrm{Hz}), 5.65(\mathrm{~d}, 1 \mathrm{H}, J=2.2 \mathrm{~Hz}), 3.56-3.46(\mathrm{~m}, 1 \mathrm{H}), 2.32(\mathrm{~s}, 3 \mathrm{H}), 0.76(\mathrm{~d}, 3 \mathrm{H}, J=7.3 \mathrm{~Hz}) ;{ }^{13} \mathrm{C}$ NMR (125 MHz, $\left.\mathrm{CDCl}_{3}\right): \delta 170.4,140.8,134.6,134.0,130.4,128.0,126.2,125.4,122.3,79.3$, 37.6, 19.3, 16.5; IR (microscope, $\mathrm{cm}^{-1}$ ): 3032, 2981, 2935, 1755, 1478, 1185, 981; HRMS (EI, $\mathrm{m} / \mathrm{z}$ ) Calcd for $\mathrm{C}_{13} \mathrm{H}_{14} \mathrm{O}_{2}: 202.09938$. Found: 202.09928 . 


\section{Synthesis of cis-4-Methyl-3-methylene-5-p-tolyl-dihydro-furan-2-one (14a)}

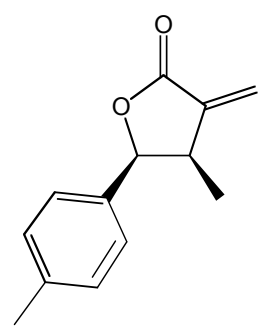

Flash chromatography (20\% EtOAc/Hexanes) yielded the product as a white solid in $48 \%$ yield. ${ }^{1} \mathrm{H}$ NMR $\left(500 \mathrm{MHz}, \mathrm{CDCl}_{3}\right): \delta 7.19-7.15(\mathrm{~m}, 2 \mathrm{H}), 7.07-7.03(\mathrm{~m}, 2 \mathrm{H}), 6.32(\mathrm{~d}, 1 \mathrm{H}, J=2.9$ $\mathrm{Hz}), 5.59$ (d, $1 \mathrm{H}, J=8.1 \mathrm{~Hz}), 5.56(\mathrm{~d}, 1 \mathrm{H}, J=2.6 \mathrm{~Hz}), 3.46-3.38(\mathrm{~m}, 1 \mathrm{H}), 2.35(\mathrm{~s}, 3 \mathrm{H}), 0.81(\mathrm{~d}$, $3 \mathrm{H}, J=7.1 \mathrm{~Hz}) ;{ }^{13} \mathrm{C} \mathrm{NMR}\left(100 \mathrm{MHz}, \mathrm{CDCl}_{3}\right): \delta 170.1,140.2,138.1,133.2,129.1,125.9,121.5$, 82.3, 39.1, 21.2, 15.4; IR $\left(\mathrm{CDCl}_{3}\right.$ cast film microscope, $\left.\mathrm{cm}^{-1}\right): 2973,1767,1247,1149,1123$, 984; HRMS (EI, m/z) Calcd for $\mathrm{C}_{13} \mathrm{H}_{14} \mathrm{O}_{2}$ : 202.09938. Found: 202.09959

\section{Synthesis of cis-4-methyl-3-methylene-5-phenyl-dihydro-furan-2-one (15a)}

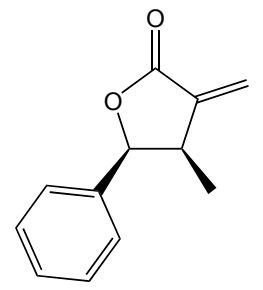

Flash chromatography (15\% EtOAc/Hexanes) yielded the product as a pale yellow solid in $67 \%$ yield. Spectral data for 15a was identical to that found in literature. ${ }^{7}$

\section{Synthesis of cis-4-Methyl-3-methylene-5-propenyl-dihydro-furan-2-one (16a)}

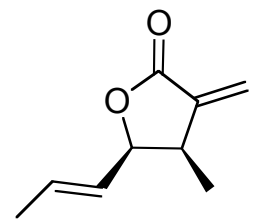

Flash chromatography (20\% EtOAc/Hexanes) yielded the product as a colorless oil in $92 \%$ yield. ${ }^{1} \mathrm{H}$ NMR (400 MHz, $\left.\mathrm{CDCl}_{3}\right): \delta 6.23(\mathrm{~d}, 1 \mathrm{H}, J=2.9 \mathrm{~Hz}), 5.82(\mathrm{dqd}, 1 \mathrm{H}, J=15.1,6.5,0.9 \mathrm{~Hz})$, $5.52(\mathrm{~d}, 1 \mathrm{H}, J=2.7 \mathrm{~Hz}), 5.37(\mathrm{ddq}, 1 \mathrm{H}, J=15.2,8.1,1.7 \mathrm{~Hz}), 4.95(\mathrm{t}, 1 \mathrm{H}, J=8.0 \mathrm{~Hz}), 3.26-$ $3.15(\mathrm{~m}, 1 \mathrm{H}), 1.76(\mathrm{ddd}, 3 \mathrm{H}, J=6.6,1.7,0.5 \mathrm{~Hz}), 1.13(\mathrm{~d}, 3 \mathrm{H}, J=7.1 \mathrm{~Hz}) ;{ }^{13} \mathrm{C} \mathrm{NMR}(100 \mathrm{MHz}$, $\mathrm{CDCl}_{3}$ ): $\delta 170.4,140.4,132.0,125.5,120.9,82.1,38.1,17.8,14.4$; IR (cast film, $\mathrm{cm}^{-1}$ ): 2970, 2934, 1763, 1451, 1156, 963; HRMS (EI, m/z) Calcd for $\mathrm{C}_{9} \mathrm{H}_{12} \mathrm{O}_{2}$ : 152.08372. Found: 152.08360 . 


\section{Synthesis of cis-5-Hept-1-ynyl-4-methyl-3-methylene-dihydro-furan-2-one (17a)}

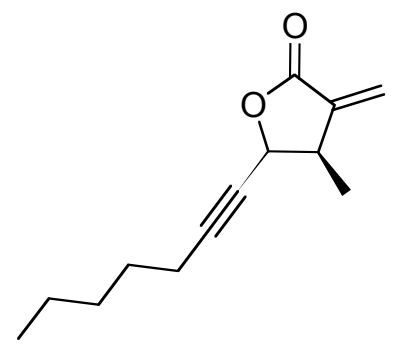

Flash chromatography (10\% EtOAc/Hexanes) yielded the product as a pale yellow oil in $51 \%$ yield. Spectral data for this compound was identical to that reported when the $E$-allylboronate and corresponding aldehyde were reacted under $\mathrm{TfOH}$ acid conditions.

Synthesis of cis-4-Methyl-3-methylene-5-phenethyl-dihydro-furan-2-one (18a)

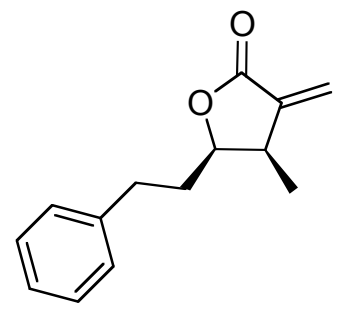

Flash chromatography (20\% EtOAc/Hexanes) yielded the product as a colorless oil in 59\% yield. Spectral data for this compound was identical to that reported when the $E$-allylboronate and corresponding aldehyde were reacted under $\mathrm{TfOH}$ acid conditions.

Synthesis of cis-5-Cyclohexyl-4-methyl-3-methylene-dihydro-furan-2-one (19a)

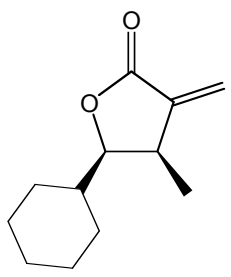

Flash chromatography (20\% EtOAc/Hexanes followed by a second column using 100\% dicholormethane) yielded the product as a colorless oil in $66 \%$ yield. Spectral data for this compound was identical to that reported when the $E$-allylboronate and corresponding aldehyde were reacted under $\mathrm{TfOH}$ acid conditions.

General procedure for the synthesis of lactones under thermal conditions followed by $p$ Toluenesulfonic acid monohydrate using the $Z$-allylboronate ${ }^{4}$

The procedure for the thermal allylboration using the $Z$-allylboronate was identical to the thermal allylboration using the $E$-allylboronate. 


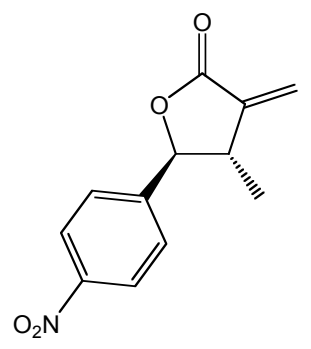

Flash chromatography (20\% EtOAc/Hexanes) yielded the product as a pale yellow solid in $61 \%$ yield. (Note: The cis diastereomer was also isolated in $17 \%$ yield). Spectral data for this compound was identical to that reported when the $Z$-allylboronate and corresponding aldehyde were reacted under $\mathrm{TfOH}$ acid conditions.

\section{Synthesis of trans-5-(4-Bromo-phenyl)-4-methyl-3-methylene-dihydro-furan-2-one (11b)}

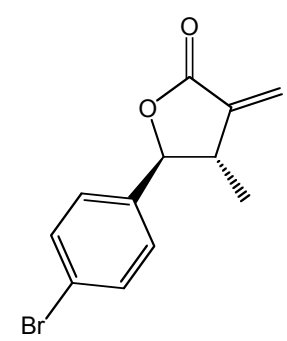

Flash chromatography (20\% EtOAc/Hexanes) yielded the product as a white solid in 53\% yield. (Note: The cis diastereomer was also isolated in 17\% yield) Spectral data for this compound was identical to that reported when the $E$-allylboronate and corresponding aldehyde were reacted under TfOH acid conditions.

\section{Synthesis of trans-5-(4-Methoxy-phenyl)-4-methyl-3-methylene-dihydro-furan-2-one (12b)}

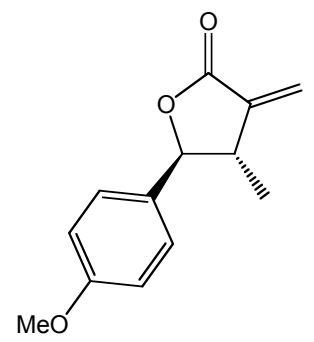

Flash chromatography (20\% EtOAc/Hexanes) yielded the product as a pale yellow solid in 57\% yield. (Note: The cis diastereomer was also isolated in 10\% yield.) Spectral data for $\mathbf{1 2 b}$ was identical to that found in literature. ${ }^{5}$ 


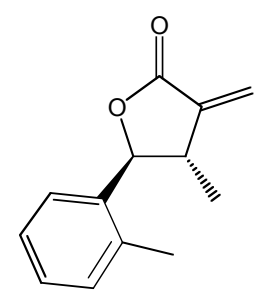

Flash chromatography (15\% EtOAc/Hexanes) yielded the product as a pale yellow oil in $49 \%$ yield. (Note: The cis diastereomer was also isolated in $8 \%$ yield.) Spectral data for this compound was identical to that reported when the $E$-allylboronate and corresponding aldehyde were reacted under $\mathrm{TfOH}$ acid conditions.

\section{Synthesis of trans-4-Methyl-3-methylene-5-p-tolyl-dihydro-furan-2-one (14b)}

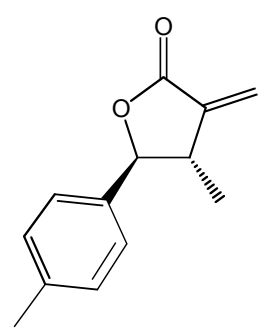

Flash chromatography (20\% EtOAc/Hexanes) yielded the product as a pale yellow oil in $71 \%$ yield. (Note: The cis diastereomer was also isolated in 3\% yield.) Spectral data for this compound was identical to that reported when the $E$-allylboronate and corresponding aldehyde were reacted under $\mathrm{TfOH}$ acid conditions.

Synthesis of trans-4-methyl-3-methylene-5-phenyl-dihydro-furan-2-one (15b)

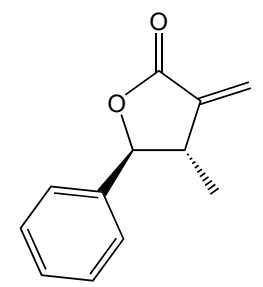

Flash chromatography (15\% EtOAc/Hexanes) yielded the product as a pale yellow solid in $66 \%$ yield. Spectral data for $\mathbf{1 5 b}$ was identical to that found in literature. ${ }^{7}$

Synthesis of trans-4-Methyl-3-methylene-5-propenyl-dihydro-furan-2-one (16b)<smiles>C=C1C(=O)OC(/C=C/C)[C@@H]1C</smiles> 
Flash chromatography (20\% EtOAc/Hexanes) yielded the product as a colorless oil in $58 \%$ yield. (Note: The cis diastereomer was also isolated in $11 \%$ yield. Compound is relatively volatile, and some product was lost during solvent removal.) Spectral data for this compound was identical to that reported when the $E$-allylboronate and corresponding aldehyde were reacted under $\mathrm{TfOH}$ acid conditions.

\section{Synthesis of trans-5-Hept-1-ynyl-4-methyl-3-methylene-dihydro-furan-2-one (17b)}

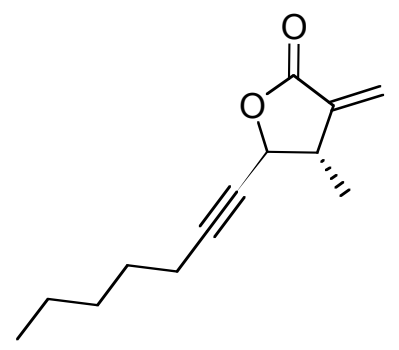

Flash chromatography (20\% EtOAc/Hexanes) yielded the product as a pale yellow oil in $62 \%$ yield. (Note: The cis diastereomer was also isolated in 12\%.) Spectral data for this compound was identical to that reported when the $Z$-allylboronate and corresponding aldehyde were reacted under $\mathrm{TfOH}$ acid conditions.

Synthesis of trans-4-Methyl-3-methylene-5-phenethyl-dihydro-furan-2-one (18b)

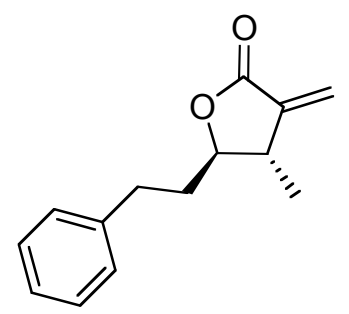

Flash chromatography (20\% EtOAc/Hexanes) yielded the product as a colorless oil in $40 \%$ yield. (Note: The cis diastereomer was also isolated in 16\% yield) Spectral data for this compound was identical to that reported when the $Z$-allylboronate and corresponding aldehyde were reacted under $\mathrm{TfOH}$ acid conditions.

Synthesis of trans-5-Cyclohexyl-4-methyl-3-methylene-dihydro-furan-2-one (19b)

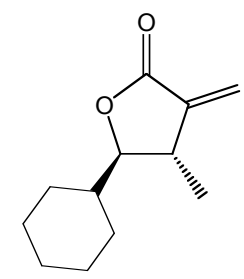

Flash chromatography (20\% EtOAc/Hexanes) yielded the product as a colorless oil in $47 \%$ yield. (Note: The cis diastereomer was also isolated in 16\% yield) Spectral data for this compound was 
identical to that reported when the $Z$-allylboronate and corresponding aldehyde were reacted under $\mathrm{TfOH}$ acid conditions.

\section{General procedure for the synthesis of butyric acid methyl esters}

A solution of the corresponding $E$ - or $Z$-allylboronate $(100 \mathrm{mg}, 0.42 \mathrm{mmol})$ and aldehyde $(0.46$ $\mathrm{mmol})$ in toluene $(0.5 \mathrm{~mL})$ was heated at $95^{\circ} \mathrm{C}$ under an $\mathrm{Ar}$ atmosphere for $42 \mathrm{~h}$. The reaction was allowed to cool to room temperature and the solvent was removed. Flash chromatography of the crude reaction mixture gave the corresponding methyl ester.

Synthesis of trans-4-Hydroxy-3-methyl-2-methylene-4-(4-nitro-phenyl)-butyric acid methyl ester (20b)<smiles>C=C(OC)[C@H](C)[C@@H](O)c1ccc([N+](=O)[O-])cc1</smiles>

Flash chromatography (20\% EtOAc/Hexanes) yielded the product as a yellow oil in 30\% yield. (Note: The corresponding trans lactone was isolated in 54\% yield and could not be completely removed from sample by chromatography. Hence, all spectra have some contamination of this lactone.) ${ }^{1} \mathrm{H} \mathrm{NMR}\left(500 \mathrm{MHz}, \mathrm{CDCl}_{3}\right): \delta 8.21-8.16(\mathrm{~m}, 2 \mathrm{H}), 7.50-7.45(\mathrm{~m}, 2 \mathrm{H}), 6.20(\mathrm{~d}, 1 \mathrm{H}, J$ $=0.8 \mathrm{~Hz}), 5.54$ (br. s, $1 \mathrm{H}), 4.86-4.81(\mathrm{~m}, 1 \mathrm{H}), 3.77(\mathrm{~s}, 3 \mathrm{H}), 3.42,(\mathrm{~d}, 1 \mathrm{H}, J=5.5 \mathrm{~Hz}), 3.08-$ $3.00(\mathrm{~m}, 1 \mathrm{H}), 1.08(\mathrm{~d}, 3 \mathrm{H}, J=7.1 \mathrm{~Hz}) ;{ }^{13} \mathrm{C} \mathrm{NMR}\left(100 \mathrm{MHz}, \mathrm{CDCl}_{3}\right): \delta 168.6,150.5,147.4$, 141.3, 127.4, 127.3, 123.4, 76.9, 52.4, 44.2, 16.3; IR $\left(\mathrm{CH}_{2} \mathrm{Cl}_{2}\right.$ cast film, $\left.\mathrm{cm}^{-1}\right): 3488,3079,2927$, 1716, 1521, 1384; HRMS (EI, m/z) Calcd for $\mathrm{C}_{12} \mathrm{H}_{12} \mathrm{NO}_{4}\left[\mathrm{M}-\mathrm{OCH}_{3}\right]^{+}:$234.07663. Found: 234.07590

Synthesis of cis-4-Hydroxy-3-methyl-2-methylene-4-(4-nitro-phenyl)-butyric acid methyl ester (20a)<smiles>C=C(OC)[C@H](C)[C@@H](O)c1ccc([N+](=O)[O-])cc1</smiles>

Flash chromatography (20\% EtOAc/Hexanes) yielded the product as a yellow oil in $69 \%$ yield. (Note: The corresponding trans lactone was isolated in $6 \%$ yield.) ${ }^{1} \mathrm{H} \mathrm{NMR}\left(400 \mathrm{MHz}, \mathrm{CDCl}_{3}\right)$ : $\delta 8.21-8.16(\mathrm{~m}, 2 \mathrm{H}), 7.56-7.51(\mathrm{~m}, 2 \mathrm{H}), 6.31(\mathrm{~d}, 1 \mathrm{H}, J=0.8 \mathrm{~Hz}), 5.59(\mathrm{t}, 1 \mathrm{H}, J=0.9 \mathrm{~Hz})$, $5.00(\mathrm{t}, 1 \mathrm{H}, J=3.4 \mathrm{~Hz}), 3.80$ (s, 3H), 3.13 (ddddd, $1 \mathrm{H}, J=7.1,7.1,7.1,3.4,1.0 \mathrm{~Hz}), 2.75$, (d, $1 \mathrm{H}, J=1.0 \mathrm{~Hz}), 0.98(\mathrm{~d}, 3 \mathrm{H}, J=7.2 \mathrm{~Hz}) ;{ }^{13} \mathrm{C} \mathrm{NMR}\left(100 \mathrm{MHz}, \mathrm{CDCl}_{3}\right): \delta 168.1,150.1,147.2$, 141.8, 127.1, 127.0, 123.3, 74.6, 52.3, 42.9, 12.0; IR (cast film microscope, $\mathrm{cm}^{-1}$ ): 3508, 2952 , 1712, 1520, 1348; HRMS (EI, m/z) Calcd for $\mathrm{C}_{12} \mathrm{H}_{12} \mathrm{NO}_{4}\left[\mathrm{M}-\mathrm{OCH}_{3}\right]^{+}:$234.07663. Found: 234.07614. 
Synthesis of cis-4-(4-Bromo-phenyl)-4-hydroxy-3-methyl-2-methylene-butyric acid methyl ester (21a)<smiles>C=C(C(C)=O)[C@@H](C)[C@@H](O)c1ccc(Br)cc1</smiles>

Flash chromatography (20\% EtOAc/Hexanes) yielded the product as a colorless oil in 56\% yield. (Note: The corresponding cis lactone was isolated in $11 \%$ yield.) ${ }^{1} \mathrm{H}$ NMR (400 MHz, $\left.\mathrm{CDCl}_{3}\right): \delta 7.46-7.42(\mathrm{~m}, 2 \mathrm{H}), 7.24-7.20(\mathrm{~m}, 2 \mathrm{H}), 6.26(\mathrm{~d}, 1 \mathrm{H}, J=0.8 \mathrm{~Hz}), 5.55-5.53(\mathrm{~m}$, $1 \mathrm{H}), 4.84$ (t, 1H, $J=3.6 \mathrm{~Hz}$ ), 3.77 (s, 3H), 3.08 (ddddd, 1H, $J=7.1,7.1,7.1,3.9,0.8 \mathrm{~Hz}$ ), 2.49, $(\mathrm{d}, 1 \mathrm{H}, J=3.1 \mathrm{~Hz}), 1.00(\mathrm{~d}, 3 \mathrm{H}, J=7.2 \mathrm{~Hz}) ;{ }^{13} \mathrm{C} \mathrm{NMR}\left(100 \mathrm{MHz}, \mathrm{CDCl}_{3}\right): \delta 168.2,142.2$, 141.6, 131.1, 128.0, 126.5, 121.0, 75.1, 52.2, 42.7, 12.6; IR (cast film microscope, $\mathrm{cm}^{-1}$ ): 3492 , 2975, 2950, 1767, 1713; HRMS (EI, m/z) Calcd for $\mathrm{C}_{13} \mathrm{H}_{15} \mathrm{O}_{2}{ }^{81} \mathrm{Br}$ : 300.01840. Found: 300.01798 .

Synthesis of cis-4-Hydroxy-4-(4-methoxy-phenyl)-3-methyl-2-methylene-butyric acid methyl ester (22a)<smiles>C=C(OC)[C@H](C)[C@@H](O)c1ccc(OC)cc1</smiles>

Flash chromatography (20\% EtOAc/Hexanes) yielded the product as a colorless oil in $24 \%$ yield. (Note: The corresponding trans lactone was isolated in 13\% yield and the corresponding cis lactone in $10 \%$ yield.) ${ }^{1} \mathrm{H}$ NMR $\left(400 \mathrm{MHz}, \mathrm{CDCl}_{3}\right): \delta 7.28-7.24(\mathrm{~m}, 2 \mathrm{H}), 6.88-6.84(\mathrm{~m}$, 2H), 6.24 (d, 1H, $J=1.0 \mathrm{~Hz}), 5.53$ (br. t, $1 \mathrm{H}, J=1.0 \mathrm{~Hz}), 4.84-4.81(\mathrm{~m}, 1 \mathrm{H}), 3.80$ (s, 3H), 3.76, (s, 3H), 3.12 (ddddd, 1H, $J=7.0,7.0,7.0,4.5,1.0 \mathrm{~Hz}$ ), 2.34, (br. s, 1H), 1.06 (d, 3H, $J=7.1$ $\mathrm{Hz}) ;{ }^{13} \mathrm{C}$ NMR $\left(100 \mathrm{MHz}, \mathrm{CDCl}_{3}\right): \delta 168.2,158.8,142.7,134.7,127.4,126.1,113.4,75.6,55.3$, 52.1, 42.7, 13.3; IR (cast film microscope, $\mathrm{cm}^{-1}$ ): 3496, 2952, 2838, 1766, 1715, 1513, 1248; HRMS (EI, m/z) Calcd for $\mathrm{C}_{14} \mathrm{H}_{18} \mathrm{O}_{4}: 250.12051$. Found: 250.12045.

\section{Synthesis of ${ }^{18} \mathrm{O}$-labelled aldehydes}

The synthesis of ${ }^{18} \mathrm{O}$ - $p$-nitrobenzaldehyde and ${ }^{18} \mathrm{O}$ - $p$-bromobenzaldehyde was done according to literature procedures. ${ }^{8}$ Both oxygen-18 labelled aldehydes displayed identical spectroscopic characteristics when compared the corresponding unlabeled aldehyde. The percentage of labeled to unlabeled aldehyde was determined by comparing the peak intensities for the different molecular ions in the HRMS.

For ${ }^{18} \mathrm{O}$-p-nitrobenzaldehyde, the peak heights were $3.38 \%$ for $\left[\mathrm{C}_{7} \mathrm{H}_{5} \mathrm{O}_{2}{ }^{18} \mathrm{ON}\right]^{+}$and 95.57 for $\left[\mathrm{C}_{7} \mathrm{H}_{5} \mathrm{O}_{3} \mathrm{~N}\right]^{+}$. This corresponds to an oxygen-18 labeling of $3.54 \%$.

For ${ }^{18} \mathrm{O}$-p-bromobenzaldehyde, the peak heights were $2.96 \%$ for $\left[\mathrm{C}_{7} \mathrm{H}_{5}{ }^{81} \mathrm{Br}^{18} \mathrm{O}\right]^{+}$and $83.89 \%$ for $\left[\mathrm{C}_{7} \mathrm{H}_{5} \mathrm{O}^{79} \mathrm{Br}\right]^{+}$. This corresponds to an oxygen-18 labeling of $3.53 \%$. The peaks corresponding to $\left[\mathrm{C}_{7} \mathrm{H}_{5} \mathrm{O}^{81} \mathrm{Br}\right]^{+}$and $\left[\mathrm{C}_{7} \mathrm{H}_{5}{ }^{18} \mathrm{O}^{79} \mathrm{Br}\right]^{+}$were overlapping in the HRMS and gave a 
peak height of $85.61 \%$. Due to the essentially $1: 1$ isotopic ratio of ${ }^{79} \mathrm{Br}$ and ${ }^{81} \mathrm{Br}$ in nature, the peaks were compared as above.

\section{Cycle NOE values for lactones}<smiles>[R]C1([2H])OC(=O)C(=C)C1(C)C</smiles>

\begin{tabular}{|c|c|c|c|}
\hline lactone & $\begin{array}{c}\mathrm{CH}_{3}-\mathrm{R} \\
\text { correlation }\end{array}$ & $\begin{array}{c}\mathrm{CH}_{3}-\mathrm{H}^{5} \\
\text { correlation }\end{array}$ & $\begin{array}{c}\mathrm{H}^{4}-\mathrm{H}^{5} \\
\text { correlation }\end{array}$ \\
\hline 10a & --- & 0.70 & --- \\
\hline 10b & --- & 1.85 & --- \\
\hline 11a & 0.76 & 0.16 & --- \\
\hline 11b & 0.32 & 1.13 & --- \\
\hline 13a & --- & 0.28 & --- \\
\hline 13b & --- & 1.13 & --- \\
\hline 14a & 0.61 & 0.78 & --- \\
\hline $\mathbf{1 4 b}$ & 0.14 & 1.02 & --- \\
\hline $\mathbf{1 6 a}$ & 0.89 & 0.22 & --- \\
\hline $\mathbf{1 6 b}$ & 0.00 & 0.90 & --- \\
\hline $\mathbf{1 7 a}$ & --- & --- & 4.11 \\
\hline $\mathbf{1 7 b}$ & --- & --- & 0.87 \\
\hline $\mathbf{1 8 a}$ & 1.66 & 0.24 & --- \\
\hline $\mathbf{1 8 b}$ & 0.61 & 1.40 & --- \\
\hline $\mathbf{1 9 a}$ & --- & --- & 4.73 \\
\hline $\mathbf{1 9 b}$ & --- & --- & 0.71 \\
\hline Corre & & & \\
\hline
\end{tabular}

${ }^{\mathrm{a}}$ Correlation values are obtained from cycle NOE spectrum that have been normalized to the irradiated peak intensity

\section{8. ${ }^{1} \mathrm{H}$ NMR Pattern for Proton Chemical Shift of $\mathbf{H}^{4}$ and $\mathbf{H}^{5}$}<smiles>[R]C1([2H])OC(=O)C(=C)C1(C)C</smiles>

\begin{tabular}{|l|l|l|l|}
\hline lactone & stereochemistry & $\begin{array}{l}\mathrm{H}^{4} \text { chemical } \\
\text { shift }^{\mathrm{a}}(\mathrm{ppm})\end{array}$ & $\begin{array}{l}\mathrm{H}^{5} \text { chemical } \\
\text { shift }^{\mathrm{a}}(\mathrm{ppm})\end{array}$ \\
\hline $\mathbf{1 0 a}$ & cis & $3.57-3.47$ & 5.70 \\
\hline $\mathbf{1 0 b}$ & trans & $2.96-2.86$ & 5.02 \\
\hline
\end{tabular}




\begin{tabular}{|l|l|l|l|}
\hline $\mathbf{1 1 a}$ & cis & $3.50-3.40$ & 5.59 \\
\hline $\mathbf{1 1 b}$ & trans & $2.94-2.86$ & 4.87 \\
\hline $\mathbf{1 2 a}$ & cis & $3.44-3.35$ & 5.57 \\
\hline $\mathbf{1 2 b}$ & trans & $2.97-2.91$ & 4.84 \\
\hline $\mathbf{1 3 a}$ & cis & $3.56-3.46$ & 5.83 \\
\hline $\mathbf{1 3 b}$ & trans & $3.10-3.02$ & 5.23 \\
\hline $\mathbf{1 4 a}$ & cis & $3.46-3.38$ & 5.59 \\
\hline $\mathbf{1 4 b}$ & trans & $2.99-2.90$ & 4.87 \\
\hline $\mathbf{1 5 a}$ & cis & $3.46-3.40$ & 5.60 \\
\hline $\mathbf{1 5 b}$ & trans & $2.85-2.90$ & 4.84 \\
\hline $\mathbf{1 6 a}$ & cis & $3.26-3.15$ & 4.95 \\
\hline $\mathbf{1 6 b}$ & trans & $2.78-2.68$ & 4.30 \\
\hline $\mathbf{1 7 a}$ & cis & $3.22-3.15$ & 5.23 \\
\hline $\mathbf{1 7 b}$ & trans & $3.08-3.02$ & 4.57 \\
\hline $\mathbf{1 8 a}$ & cis & $3.23-3.12$ & $4.57-4.51$ \\
\hline $\mathbf{1 8 b}$ & trans & $2.80-2.60$ & 3.99 \\
\hline $\mathbf{1 9 a}$ & cis & $2.99-2.81$ & 3.83 \\
\hline $\mathbf{1 9 b}$ & trans & & \\
\hline $\mathbf{1 0}$ & & & \\
\hline
\end{tabular}

${ }^{a}$ Chemical shift is reported based on ppm downfield of tetramethylsilane. All ${ }^{1} \mathrm{H}$ NMR experiments were performed using $\mathrm{CDCl}_{3}$ as solvent. 
9. Copies of ${ }^{1} \mathrm{H}$ NMR and ${ }^{13} \mathrm{C}$ NMR

cis-4-Methyl-3-methylene-5-(4-nitro-phenyl)-dihydro-furan-2-one (10a)

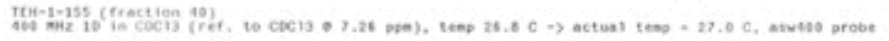

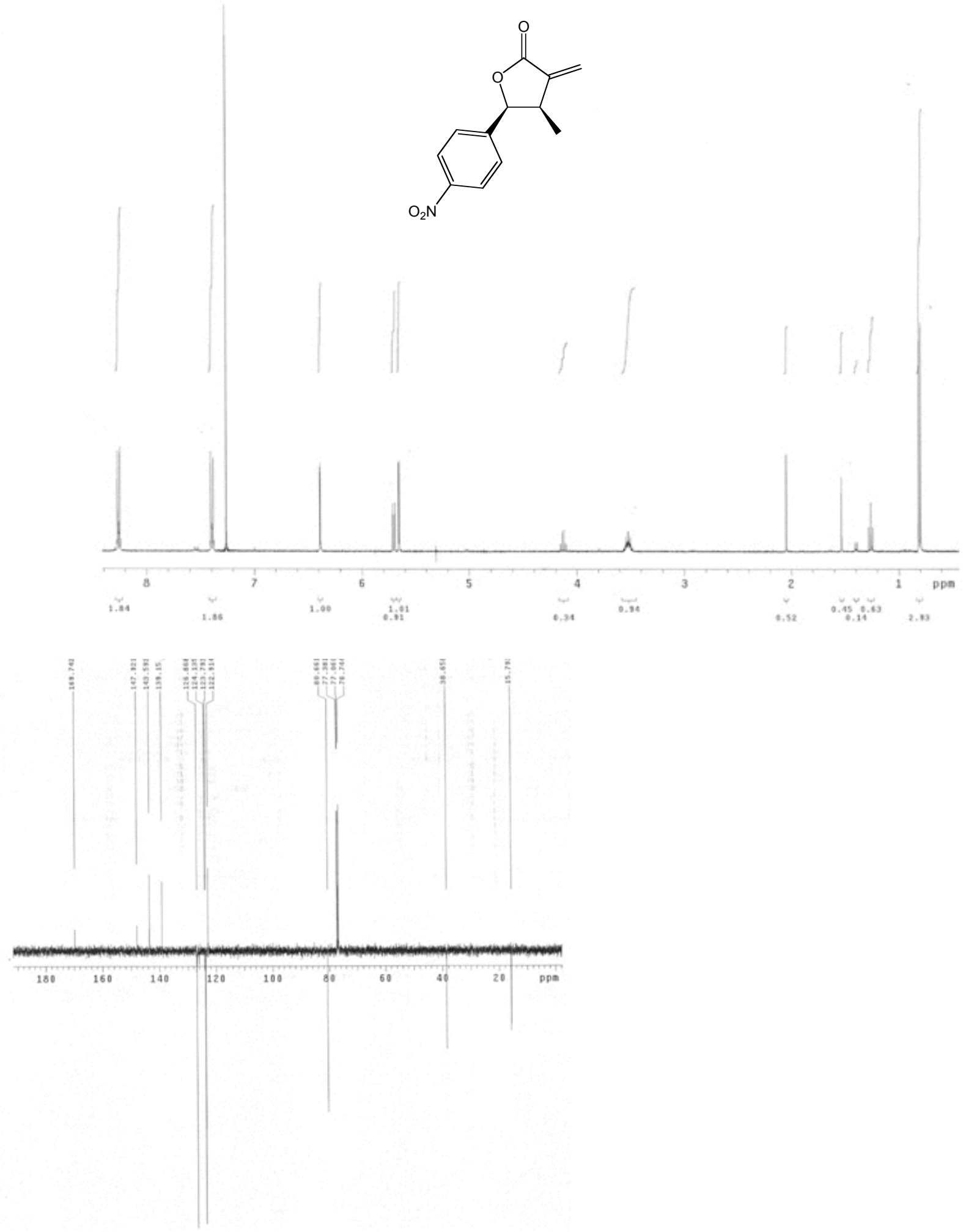


cis-5-(4-Bromo-phenyl)-4-methyl-3-methylene-dihydro-furan-2-one (11a)

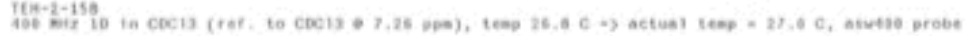
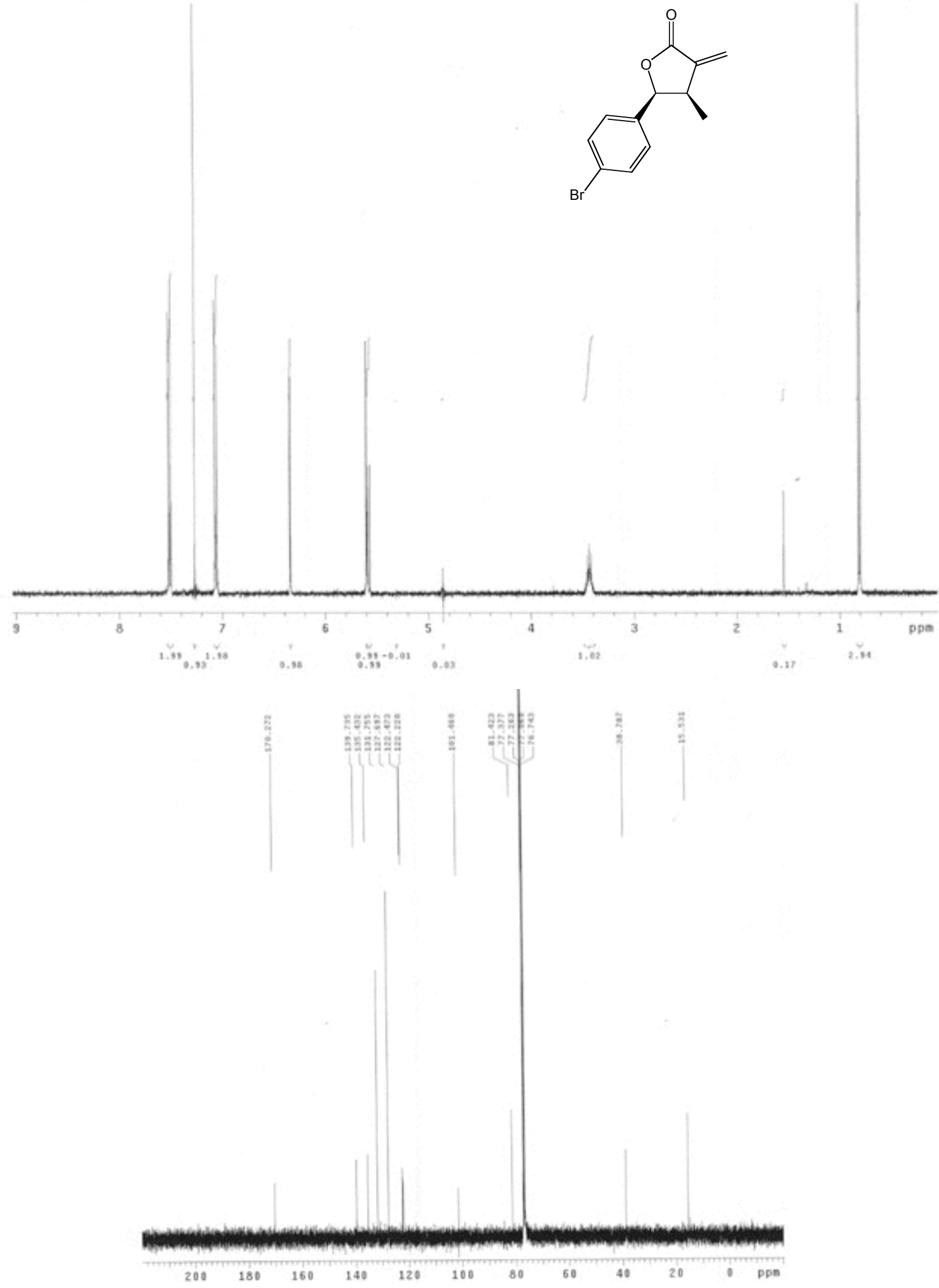
cis-4-Methyl-3-methylene-5-o-tolyl-dihydro-furan-2-one (13a)
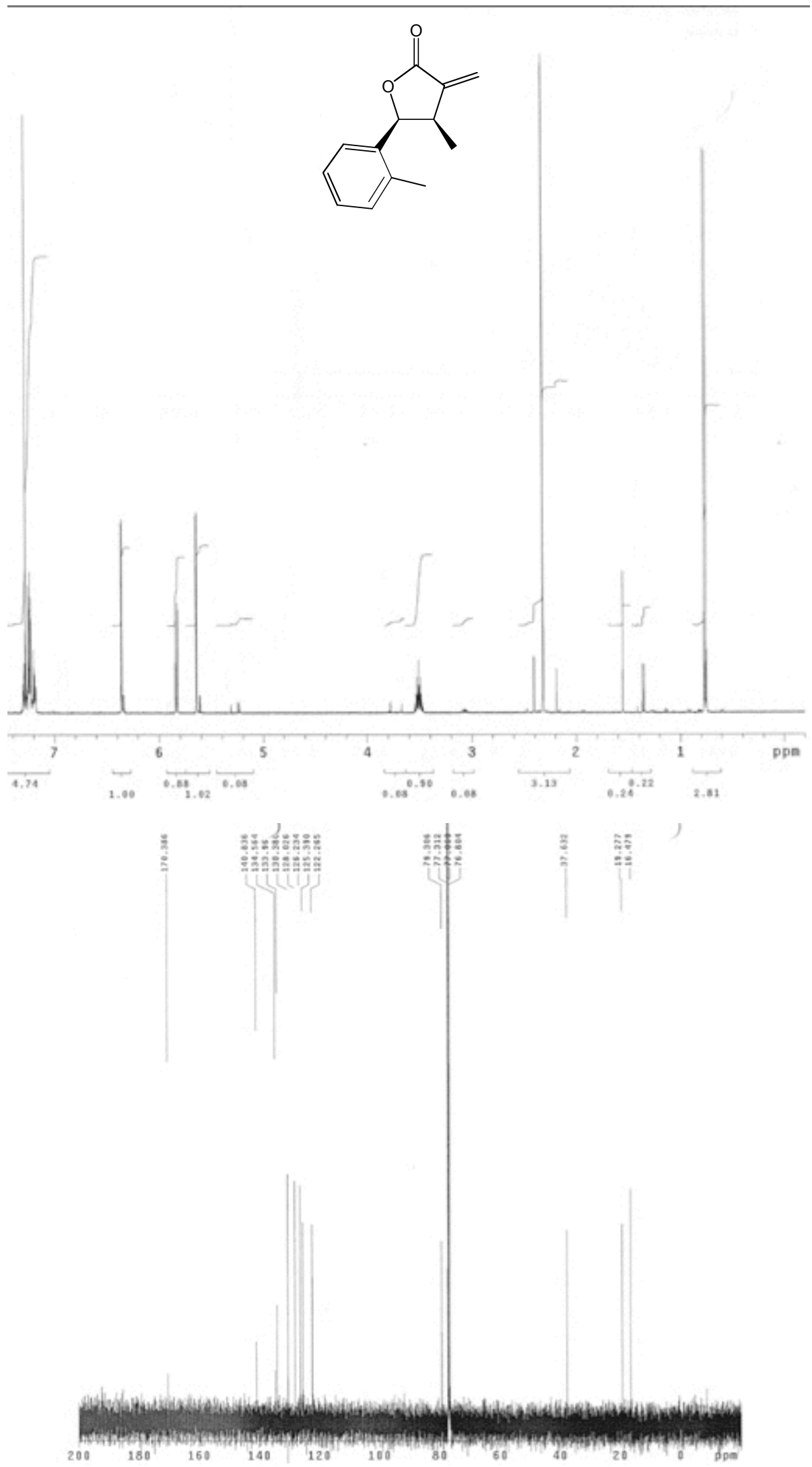
cis-4-Methyl-3-methylene-5-p-tolyl-dihydro-furan-2-one (14a)
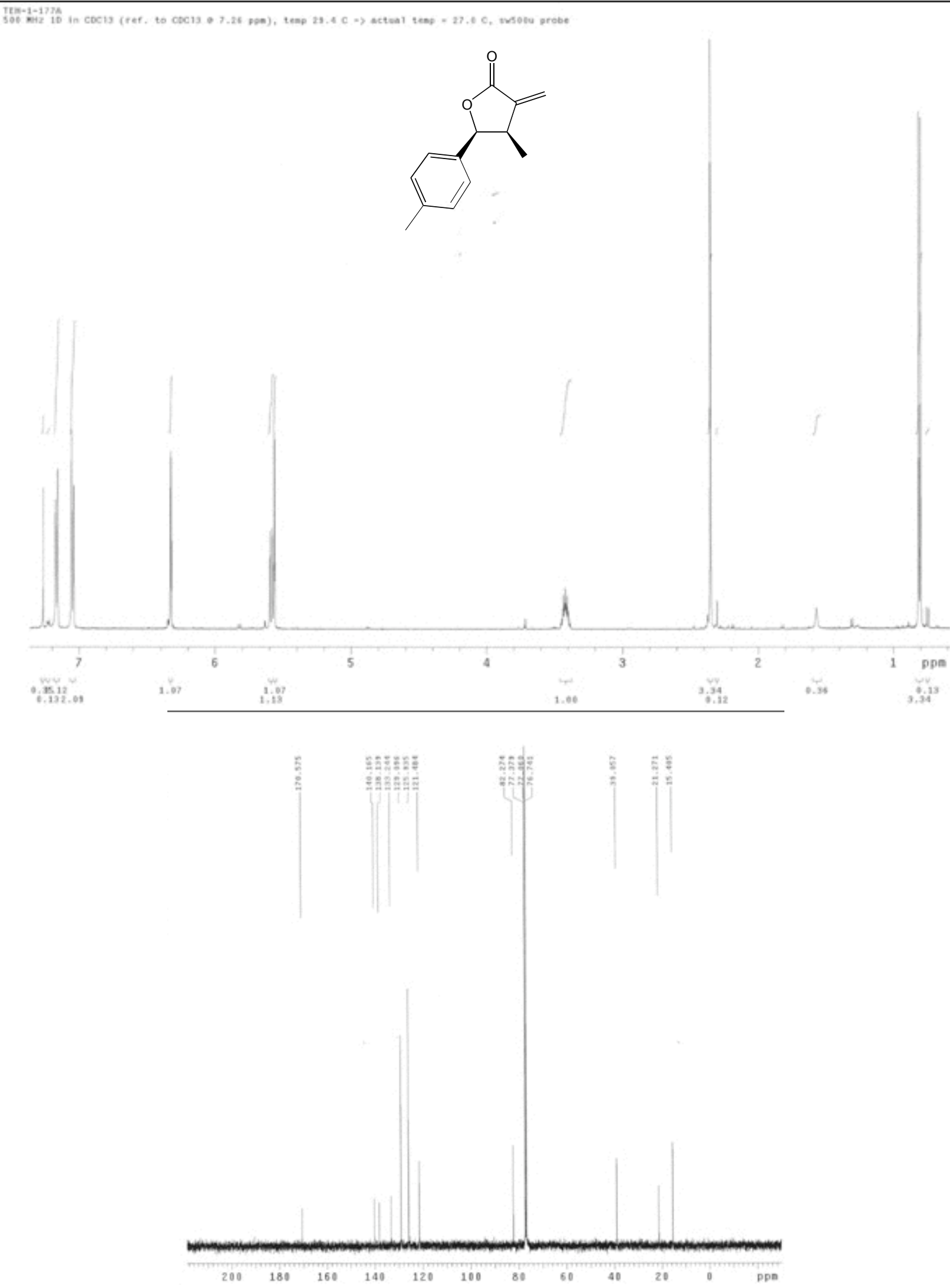
cis-4-Methyl-3-methylene-5-propenyl-dihydro-furan-2-one (16a)

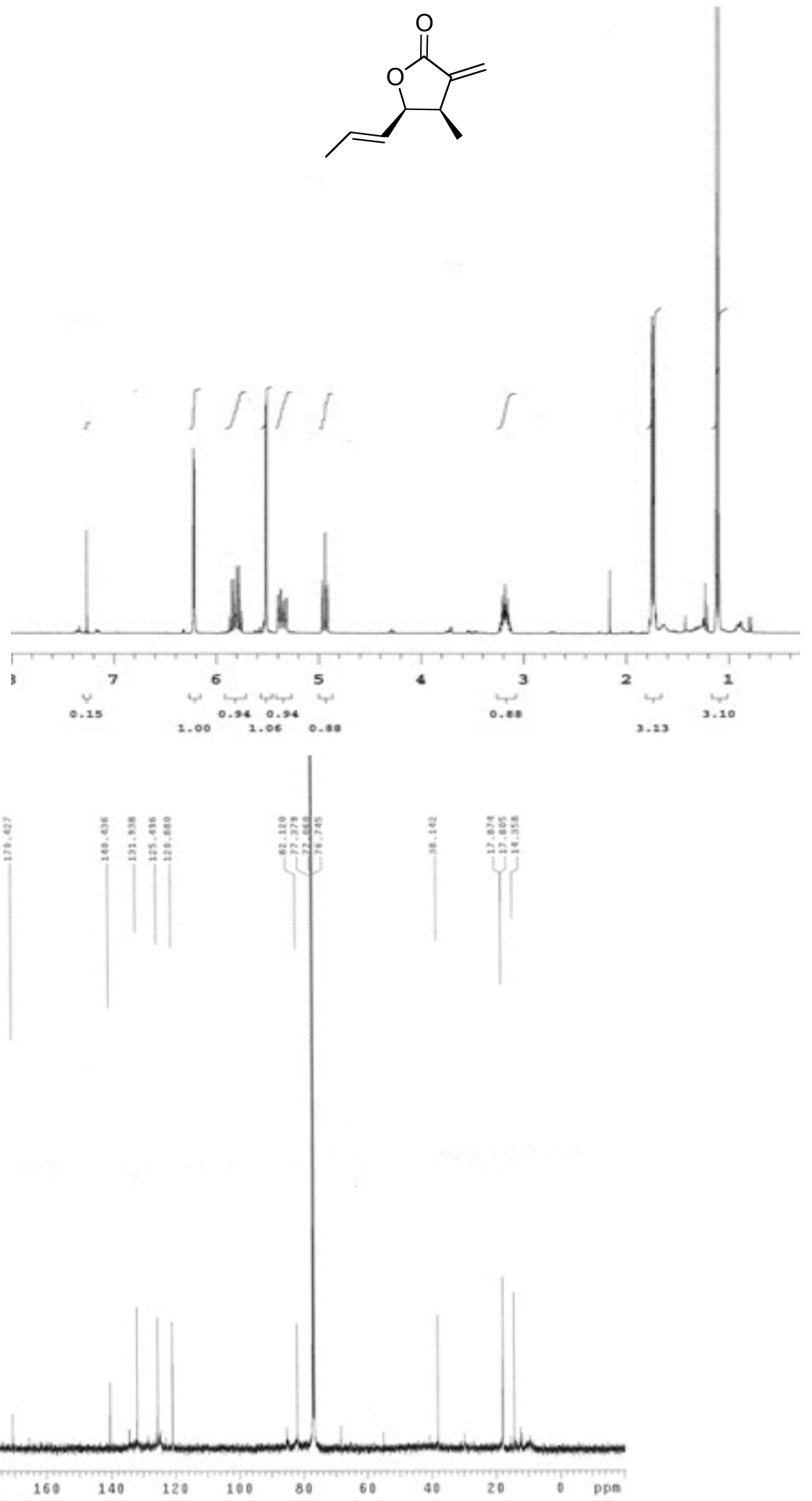


cis-5-Hept-1-ynyl-4-methyl-3-methylene-dihydro-furan-2-one (17a)
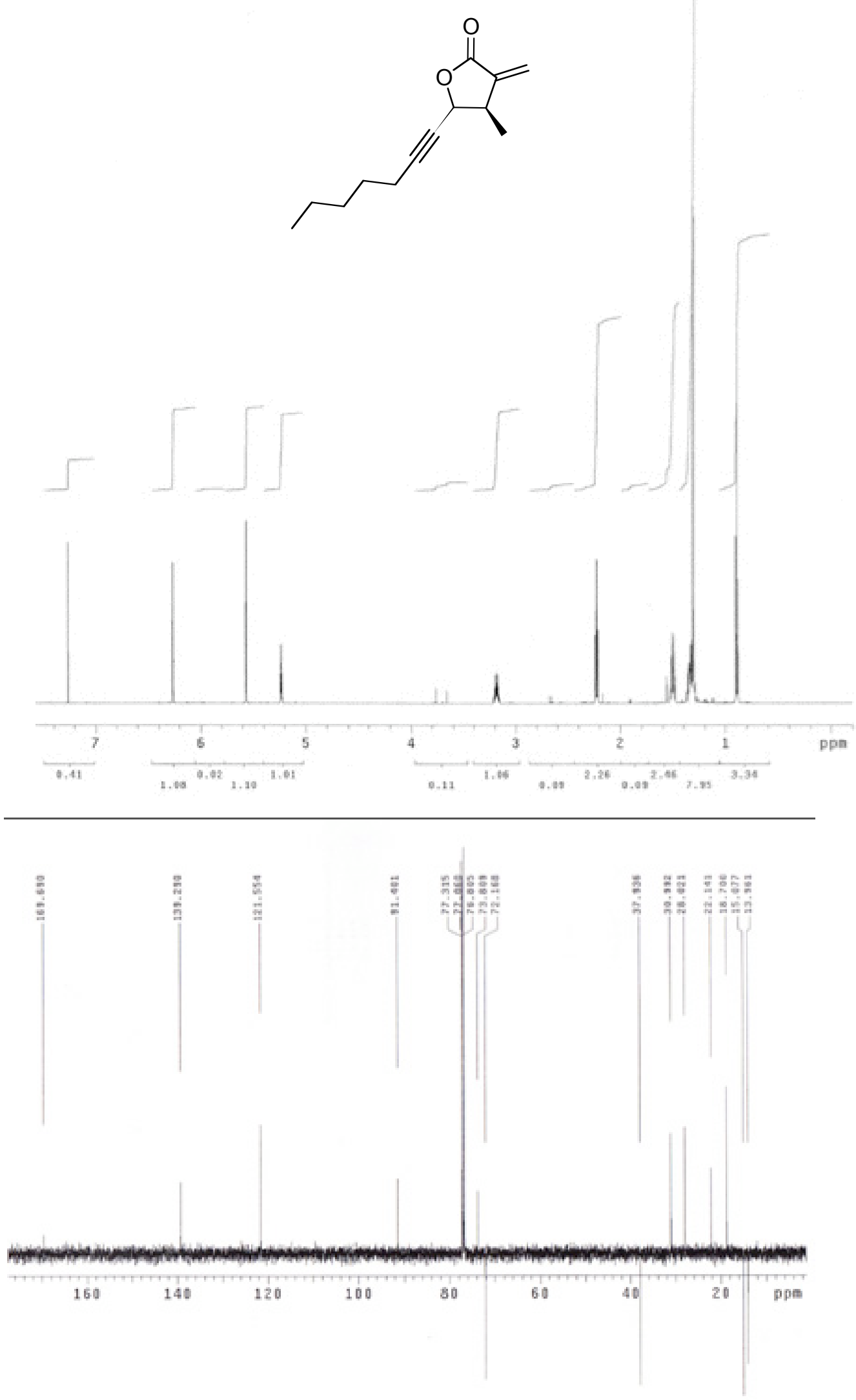
cis-4-Methyl-3-methylene-5-phenethyl-dihydro-furan-2-one (18a)
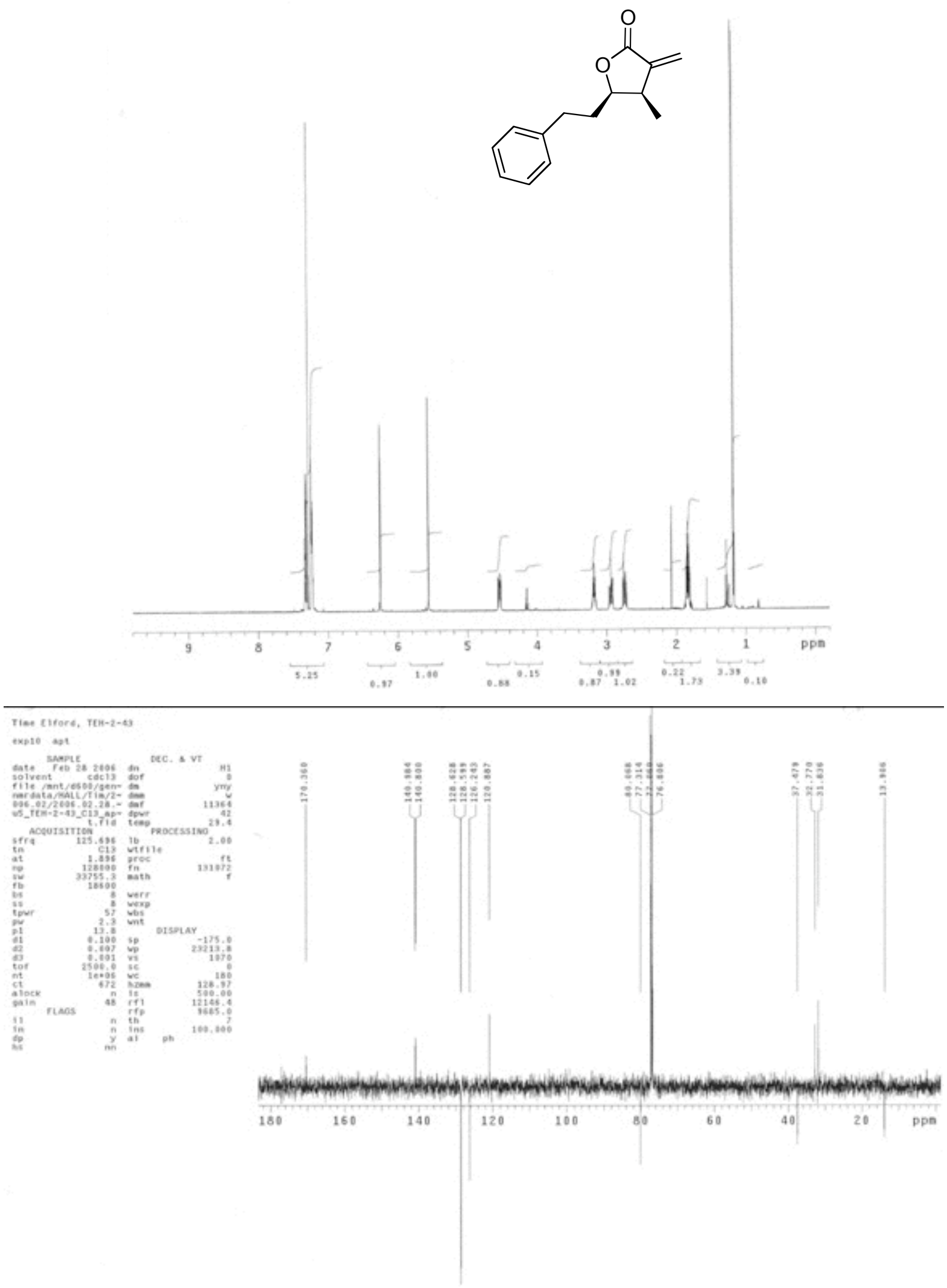
cis-5-Cyclohexyl-4-methyl-3-methylene-dihydro-furan-2-one (19a)
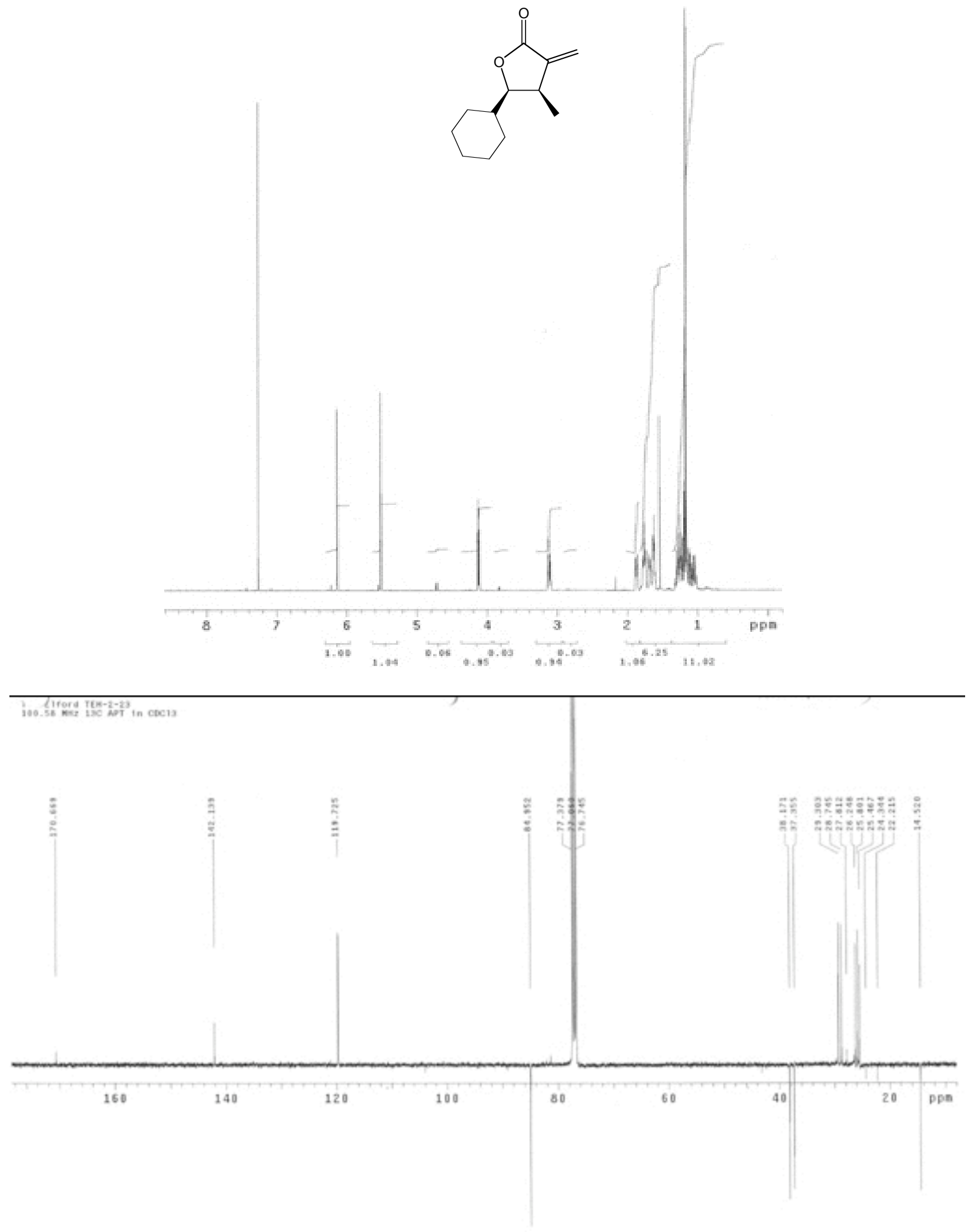
trans-4-Methyl-3-methylene-5-(4-nitro-phenyl)-dihydro-furan-2-one (10b)

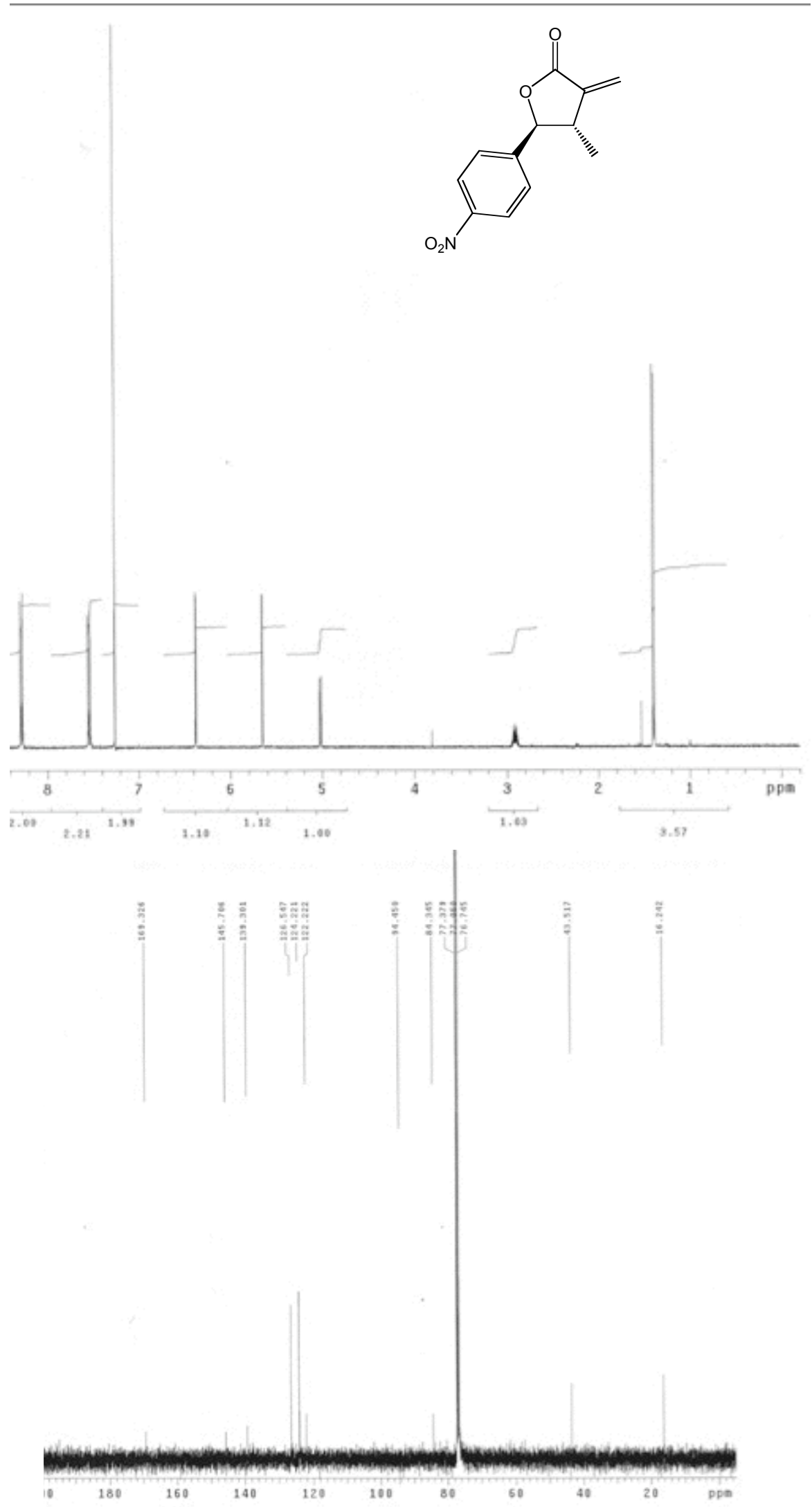


trans-5-(4-Bromo-phenyl)-4-methyl-3-methylene-dihydro-furan-2-one (11b)

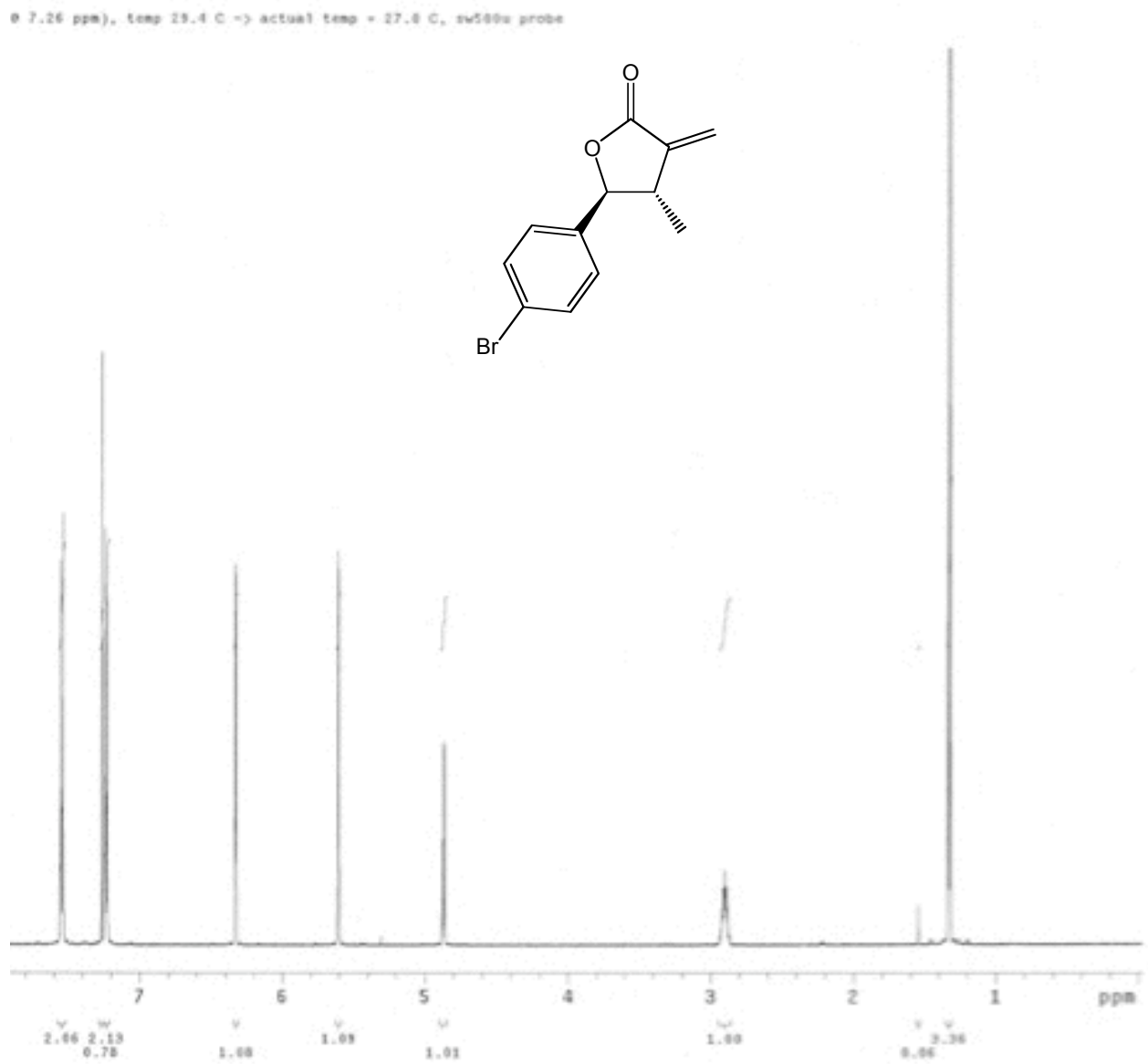

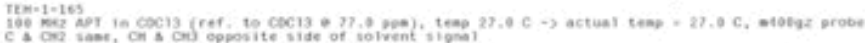

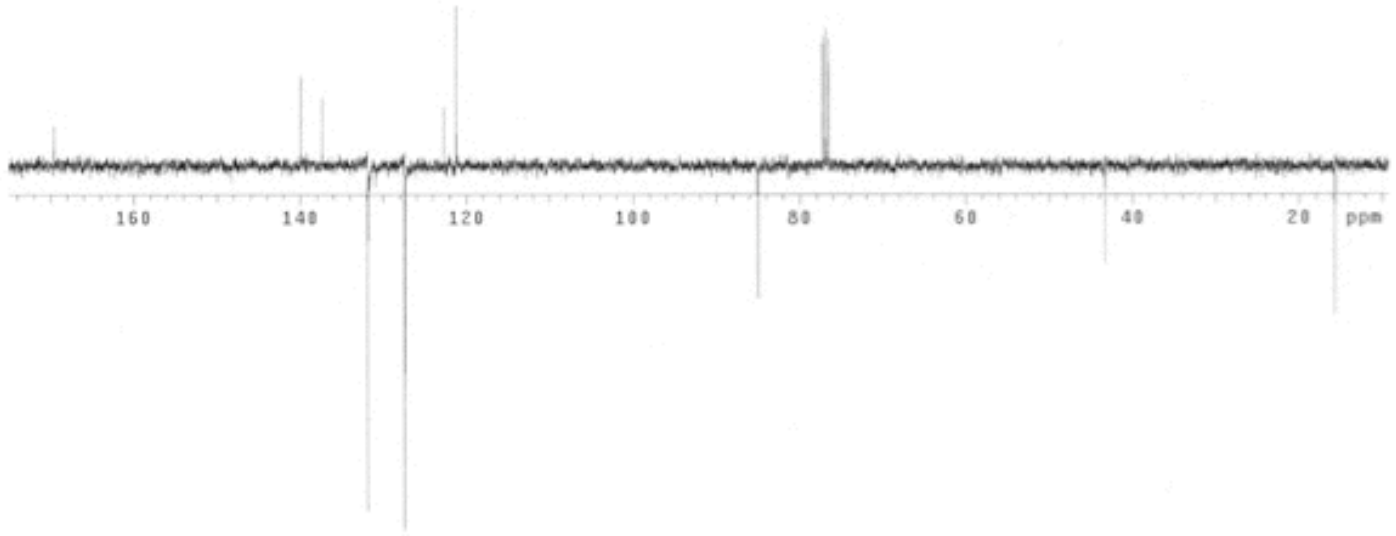


trans-4-Methyl-3-methylene-5-o-tolyl-dihydro-furan-2-one (13b)
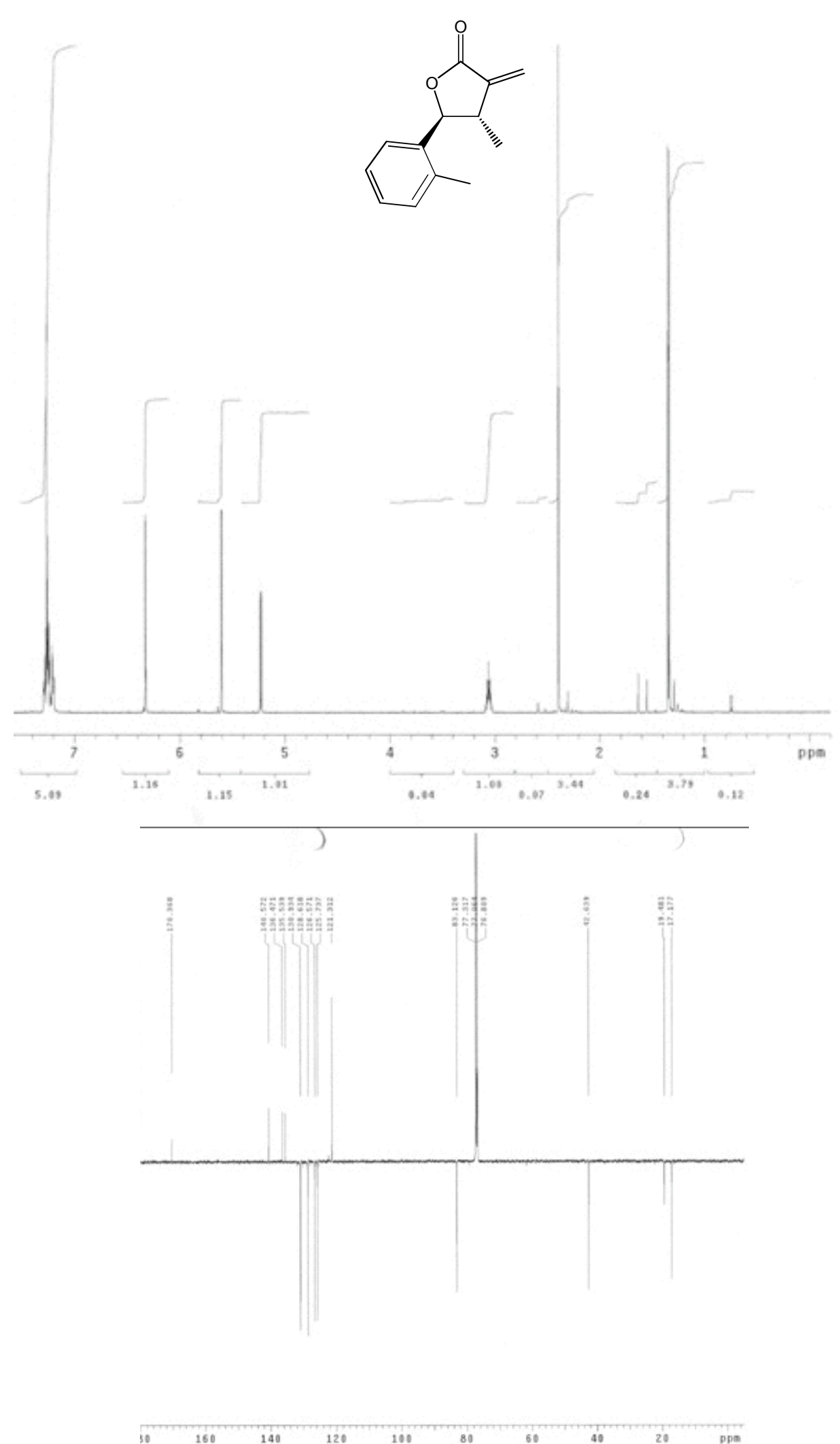

120

100

80

60

40

$20 \quad$ ppa 
trans-4-Methyl-3-methylene-5-p-tolyl-dihydro-furan-2-one (14b)

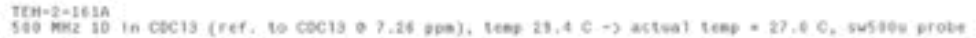

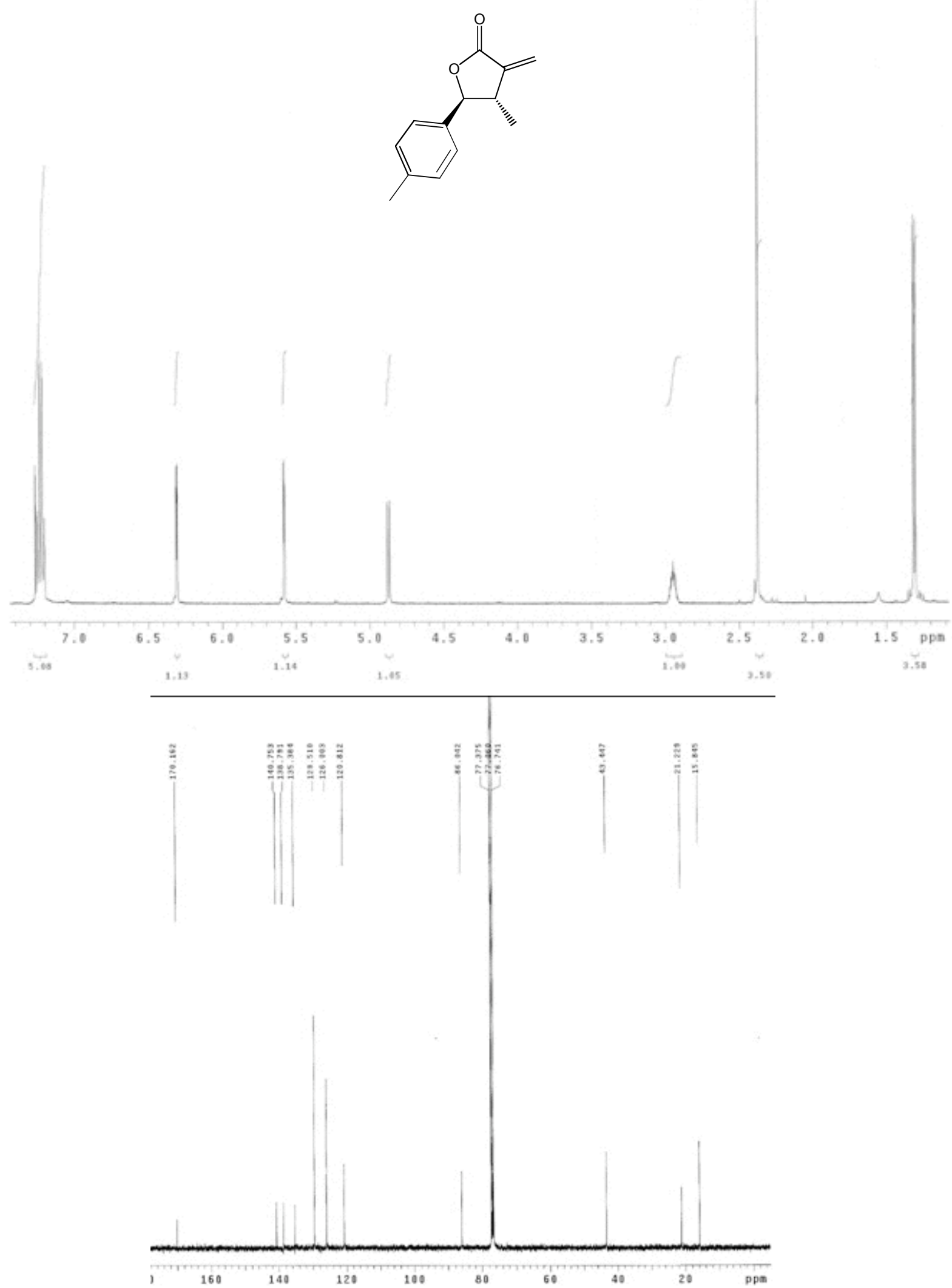


trans-4-Methyl-3-methylene-5-propenyl-dihydro-furan-2-one (16b)
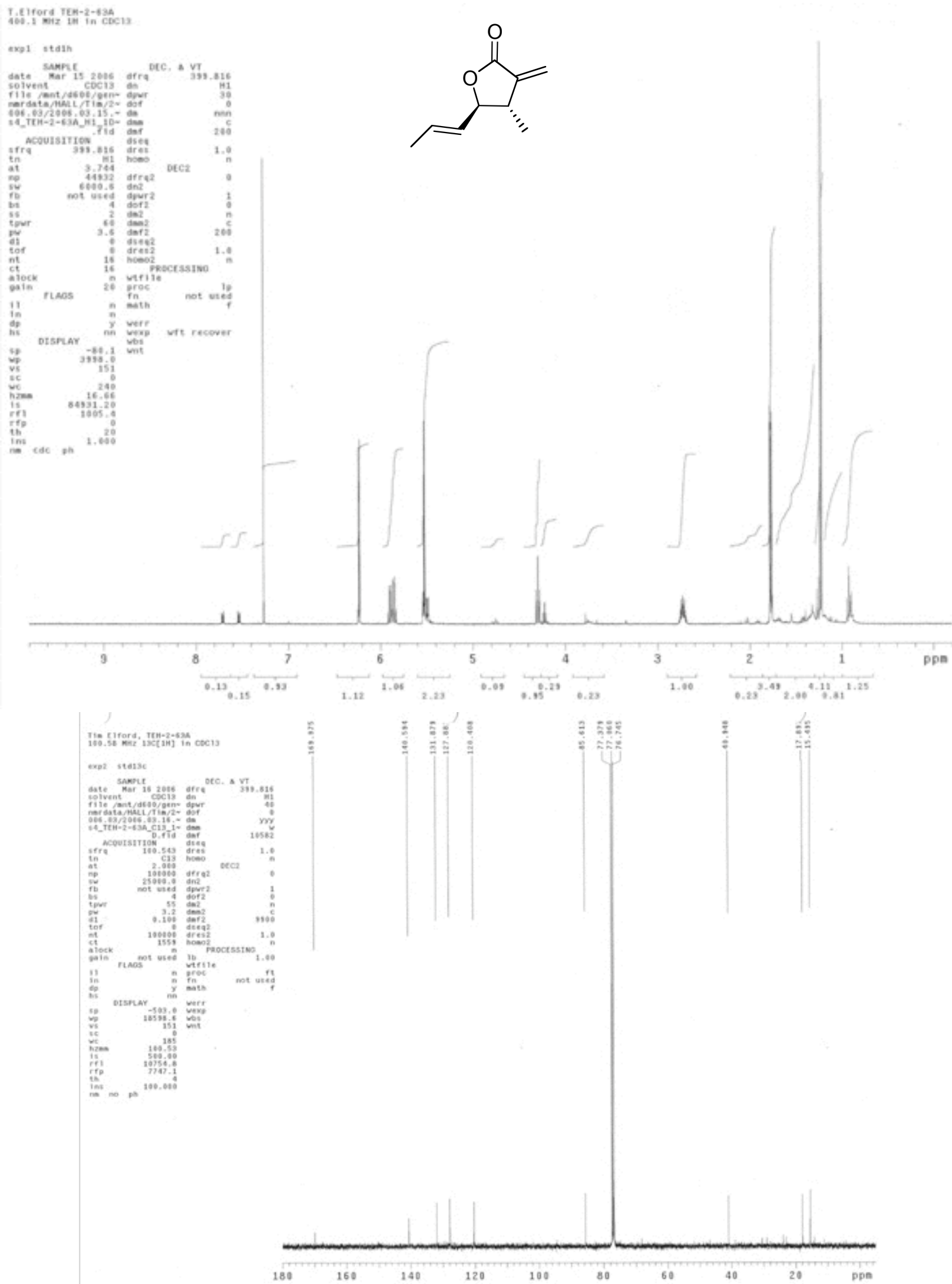
trans-5-Hept-1-ynyl-4-methyl-3-methylene-dihydro-furan-2-one (17b)
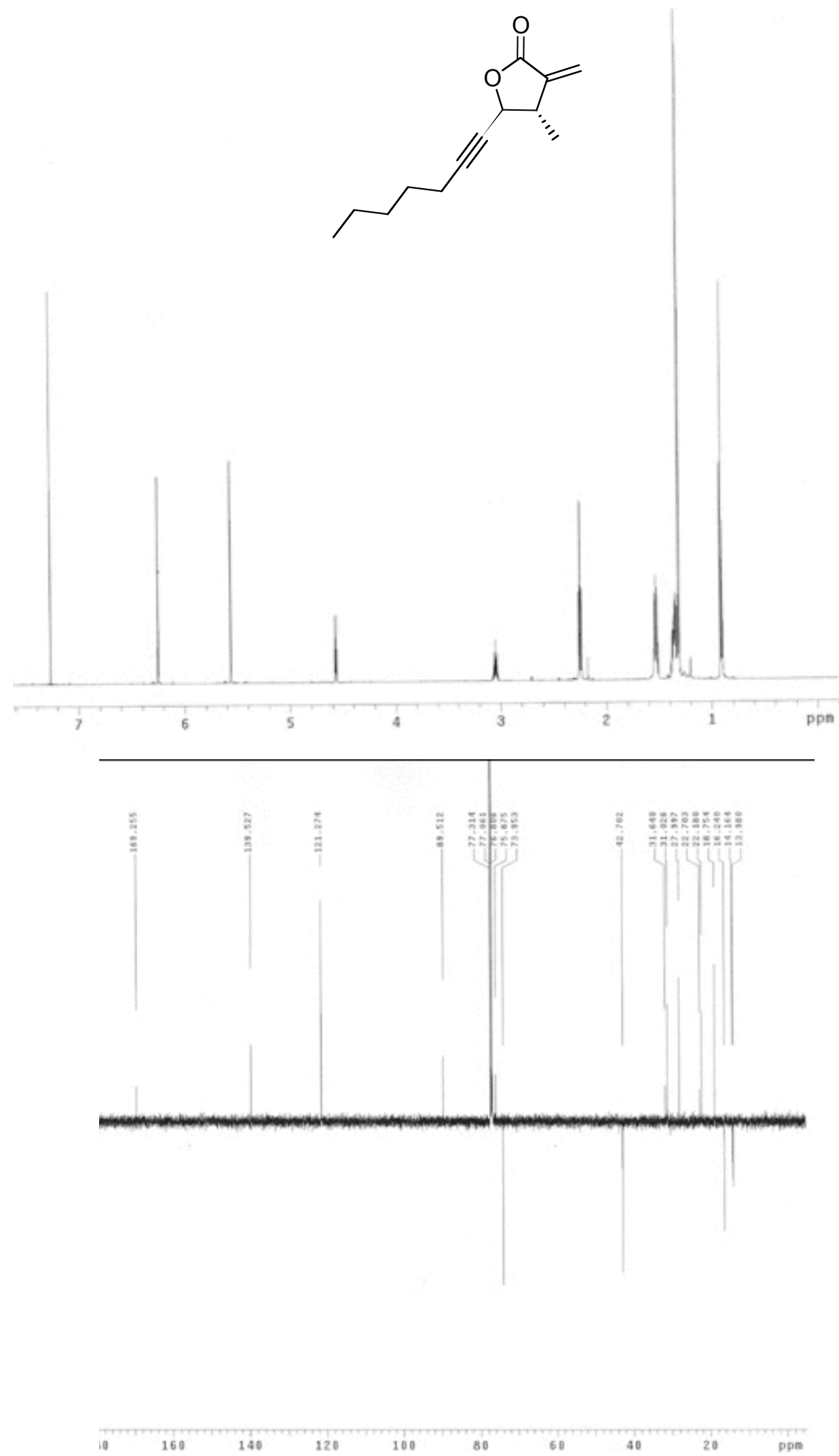
trans-4-Methyl-3-methylene-5-phenethyl-dihydro-furan-2-one (18b)
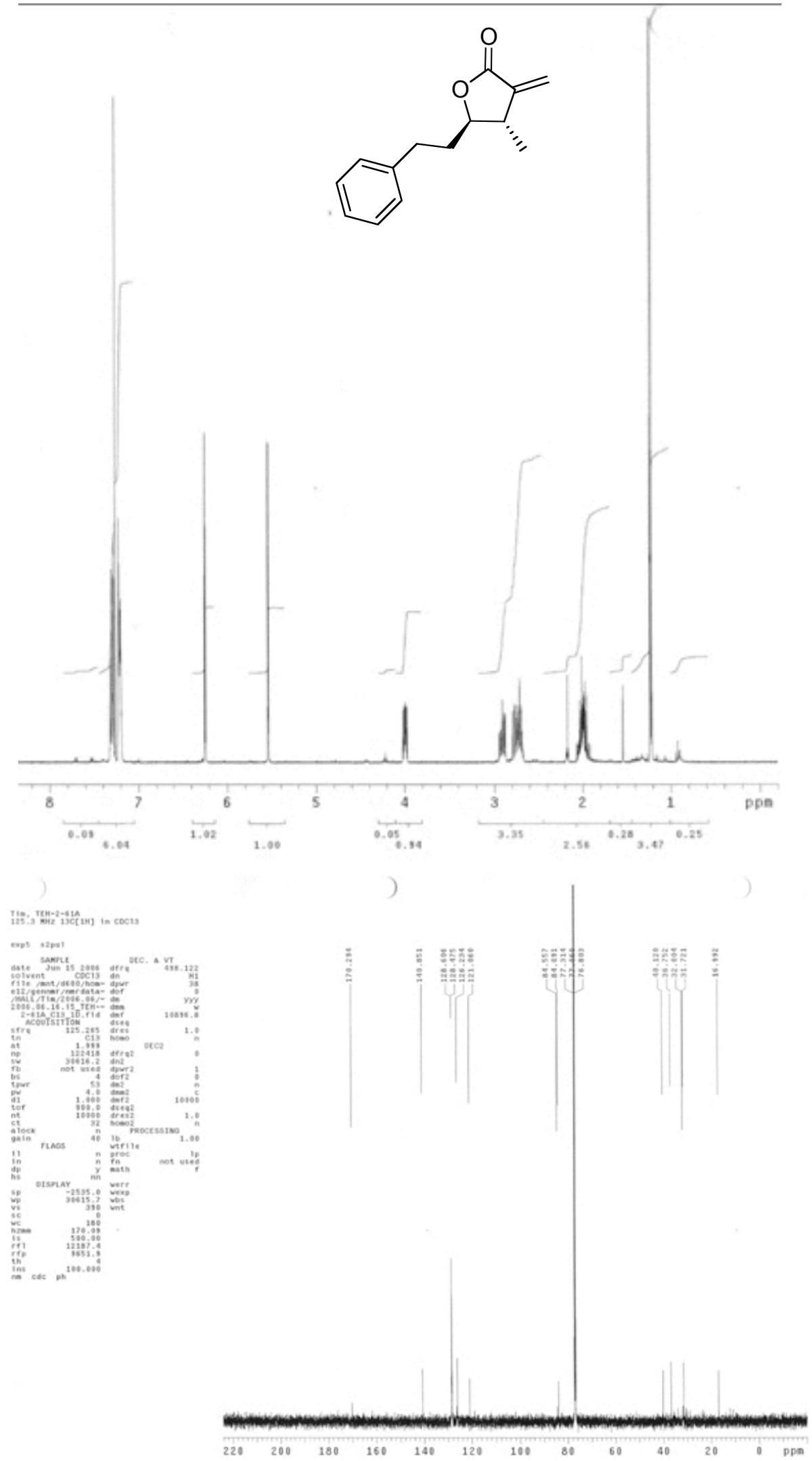
trans-5-Cyclohexyl-4-methyl-3-methylene-dihydro-furan-2-one (19b)

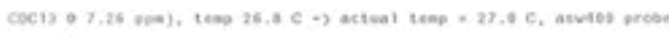

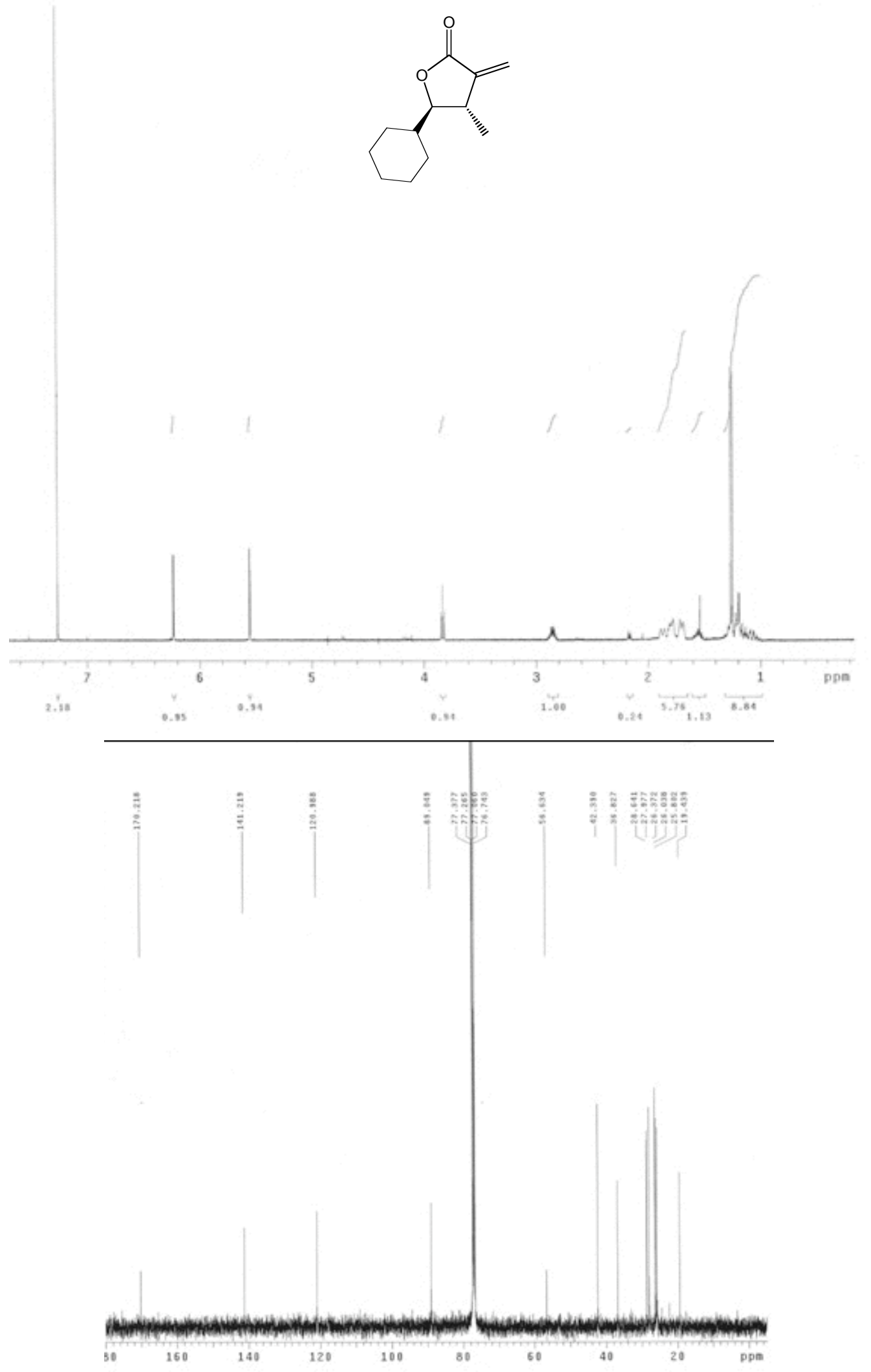

S36 
trans-4-Hydroxy-3-methyl-2-methylene-4-(4-nitro-phenyl)-butyric acid methyl ester (20b)

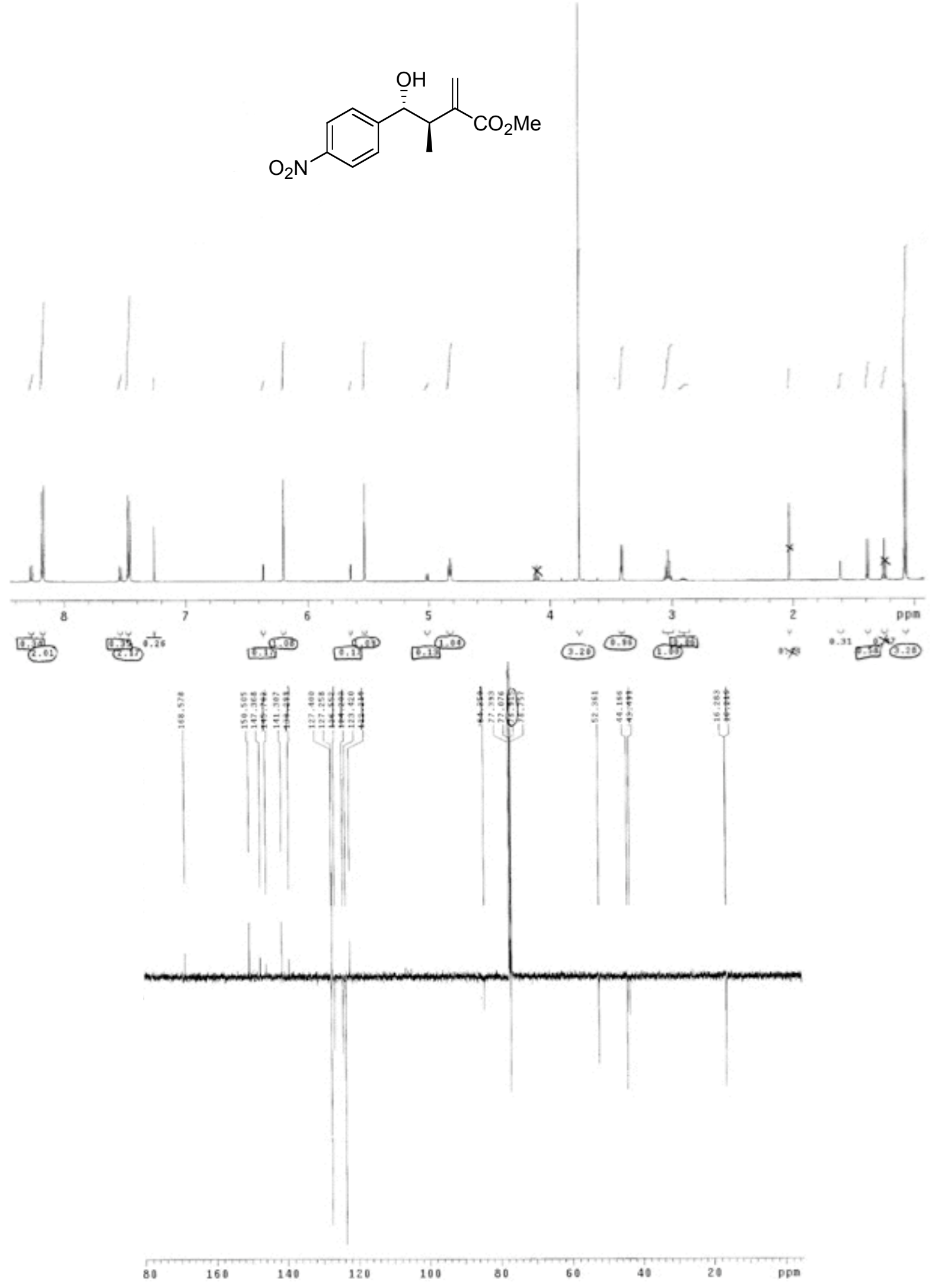


cis-4-Hydroxy-3-methyl-2-methylene-4-(4-nitro-phenyl)-butyric acid methyl ester (20a)
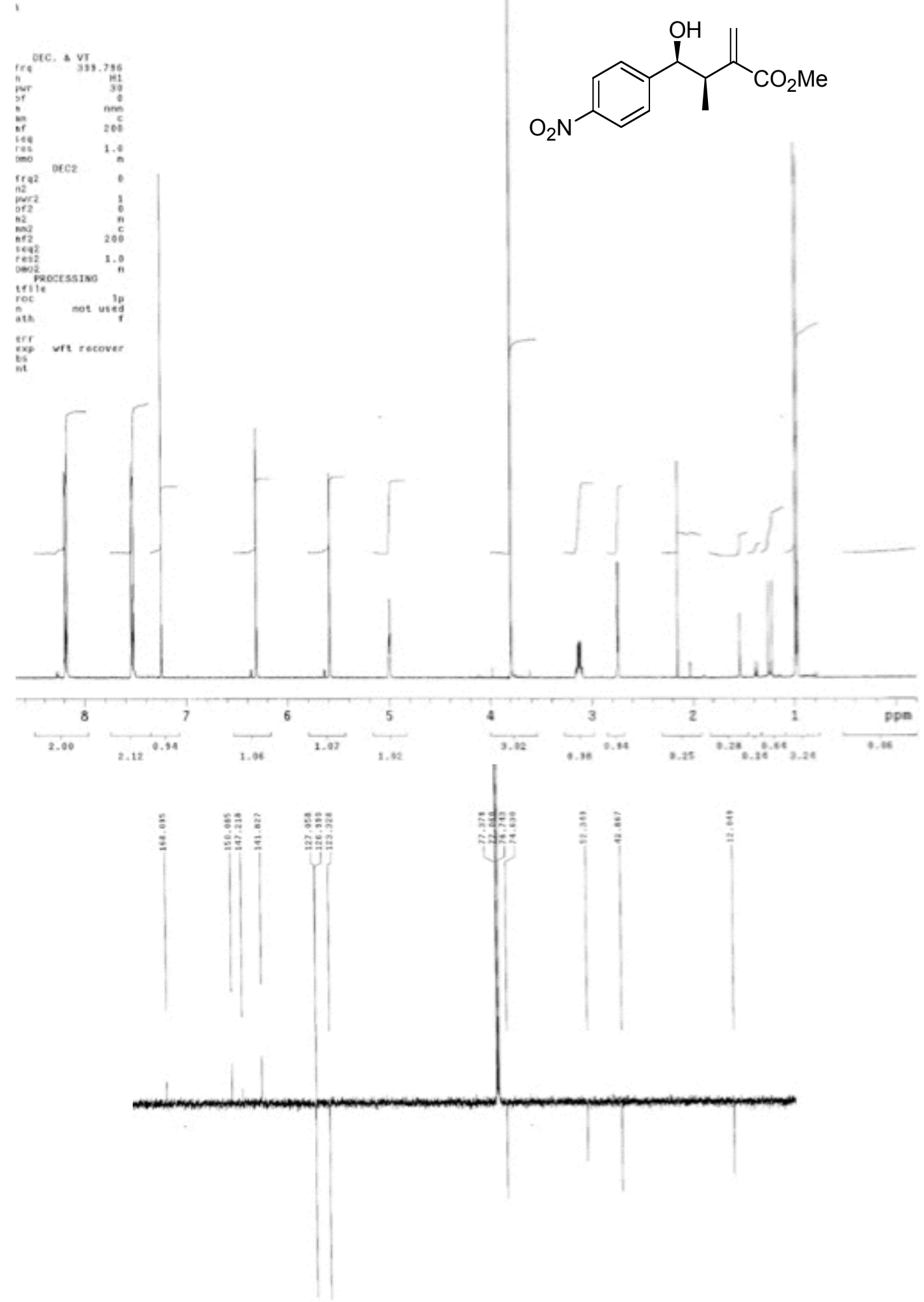

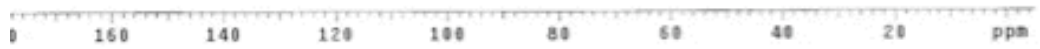


cis-4-(4-Bromo-phenyl)-4-hydroxy-3-methyl-2-methylene-butyric acid methyl ester (21a)
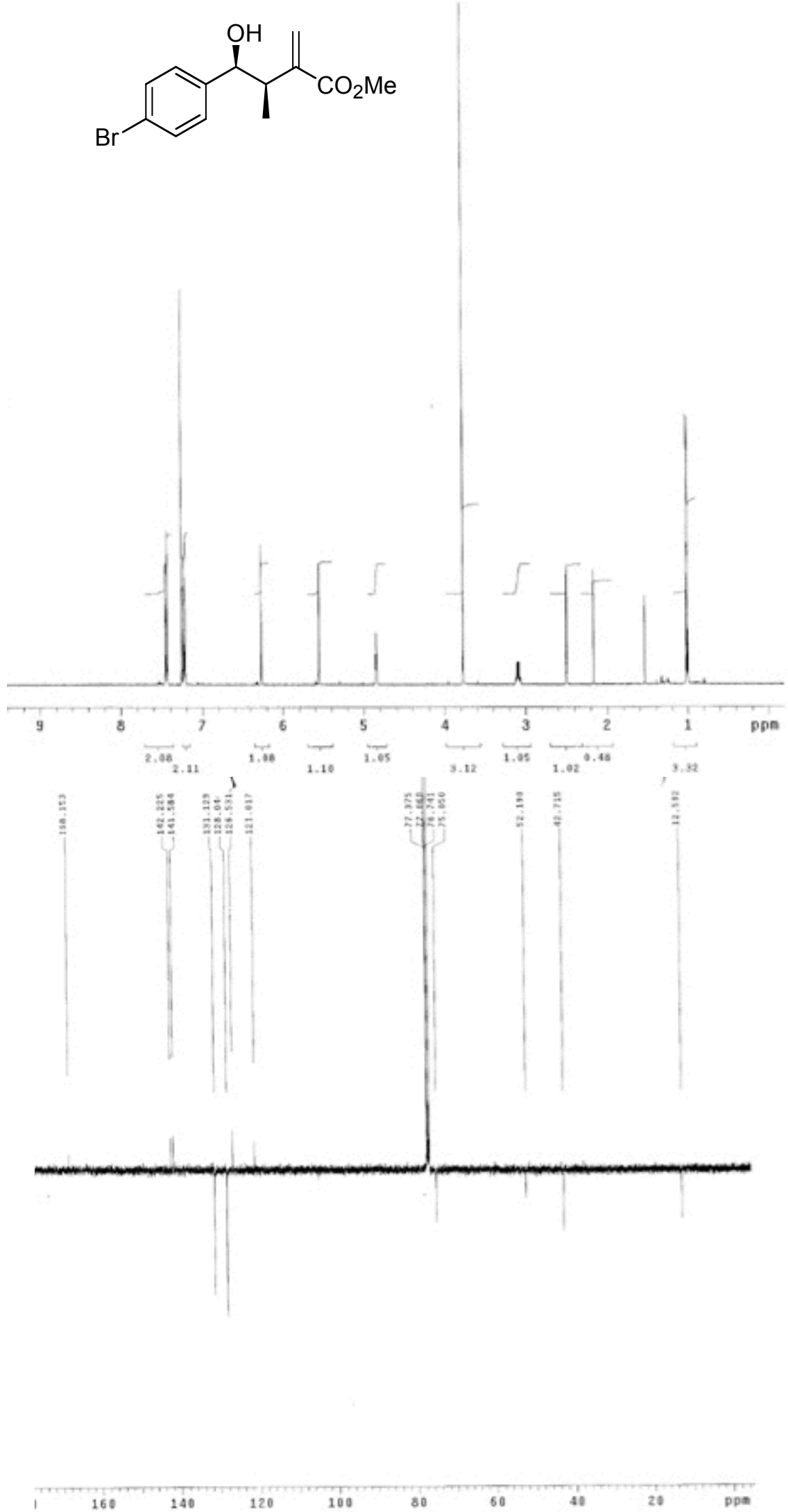
cis-4-Hydroxy-4-(4-methoxy-phenyl)-3-methyl-2-methylene-butyric acid methyl ester (22a)
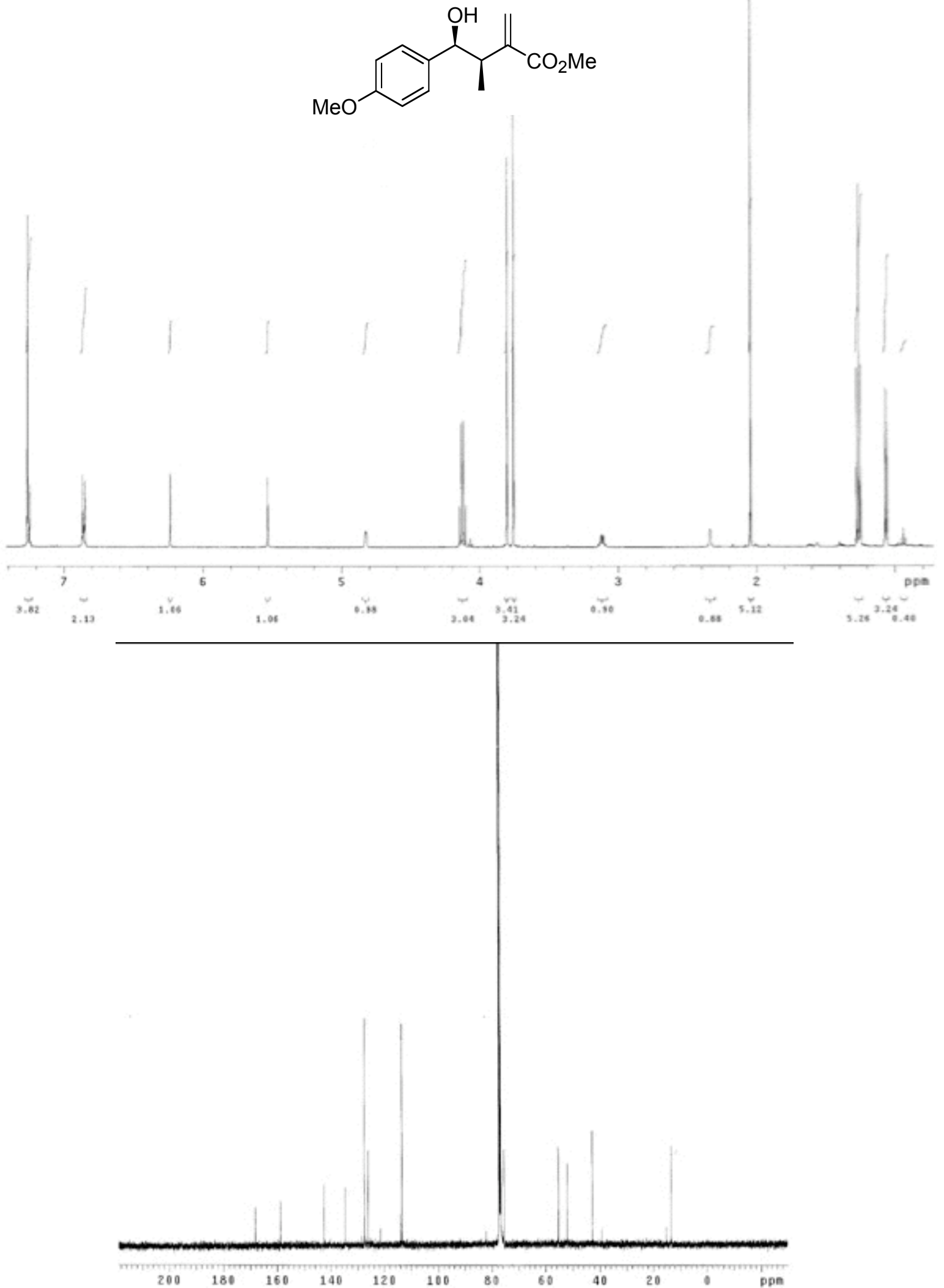
cis-4-(4-Bromo-phenyl)-3-methyl-2-methylene-4-(4,4,5,5-tetramethyl-[1,3,2] dioxaborolan2-yloxy-butyric acid methyl ester (23)

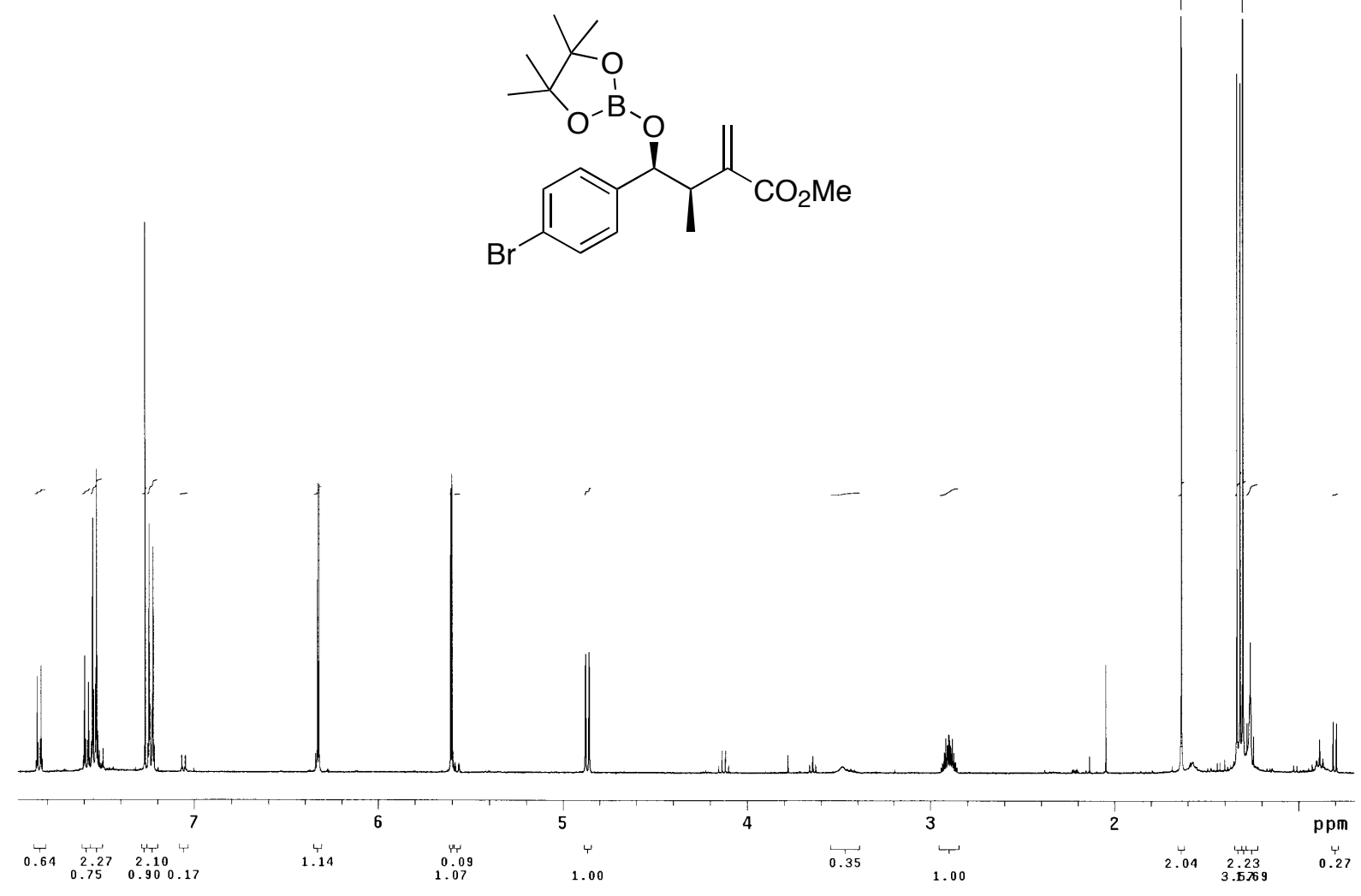


Isopropyl (2E)-2-[4,4,5,5-tetramethyl-1,3,2-dioxaborolan-2-yl)methyl]-but-2-enoate ( $E$-26)
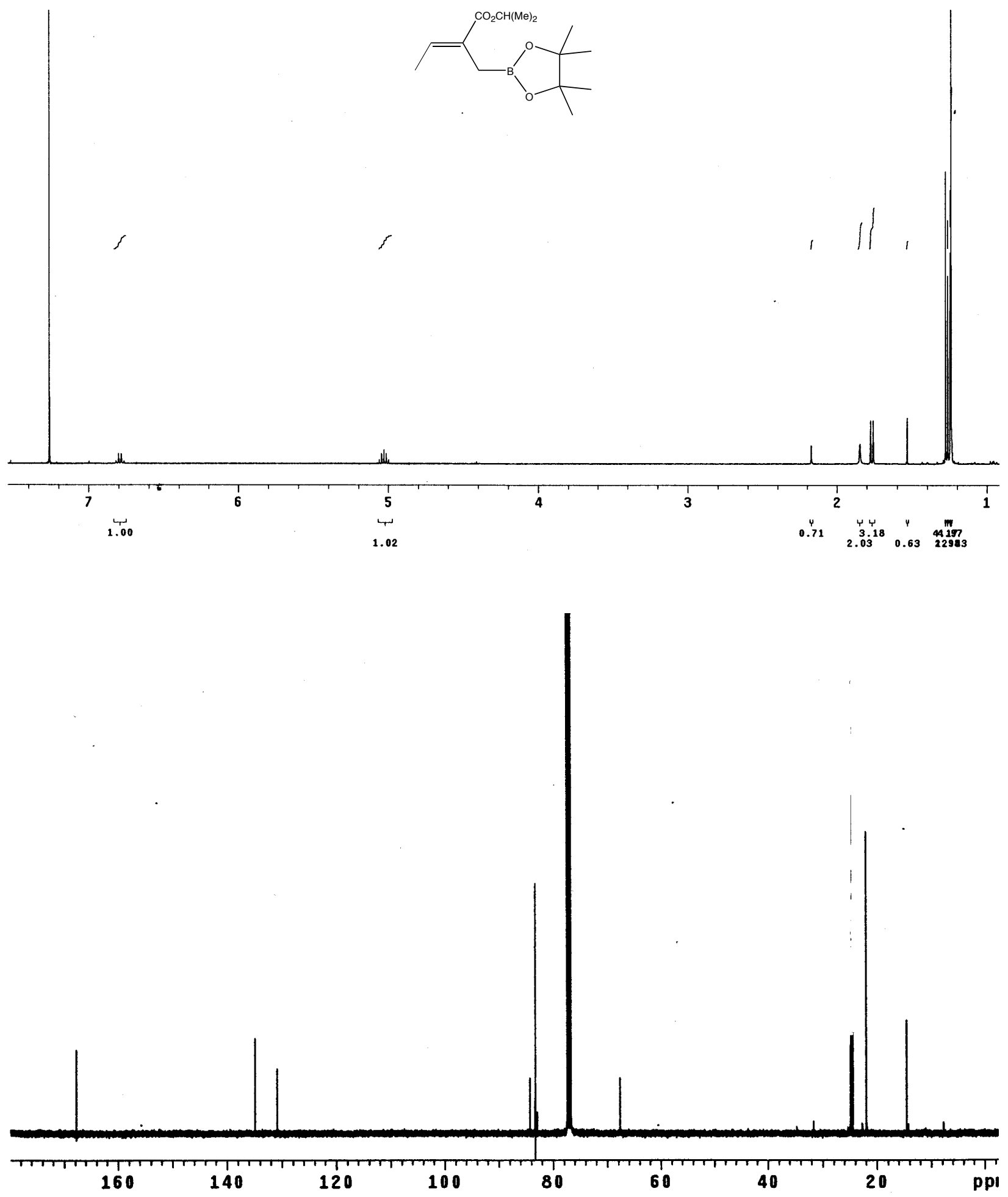

S42 
${ }^{1}$ Taylor, R. K.; Casy, G. In Organocopper Reagents: A Practical Approach; Taylor, R. K., Ed.; Oxford University Press: New York, 1994, pp 53-72.

${ }^{2}$ Phillion, D. P.; Neubauer, R.; Andrew, S. S. J. Org. Chem. 1986, 51, 1610-1612.

${ }^{3}$ Whiting, A. Tetrahedron Lett. 1991, 32, 1503-1506.

${ }^{4}$ Yu, S.H.; Ferguson, M.J.; McDonald, R.; Hall, D.G. J. Am. Chem. Soc. 2005, 127, 1280812809.

${ }^{5}$ Jung, M.E. and Buszek, K.R. J. Am. Chem. Soc. 1988, 110, 3965-3969

${ }^{6}$ Csuk, R.; Schroder, C.; Hutter, S.; Mohr, K. Tetrahedron: Asymmetry, 1997, 8(9), 1411-1429.

${ }^{7}$ Lin, S.H.; Chen, C.C.; Vong, W.J.; Liu, R.S.; Organometallics, 1995, 14, 1619-1625.

${ }^{8}$ Baraniak, J and Wojciech, J. S. J. Chem. Soc. Perkin Trans. 1 1987, 8, 1645-1656. 